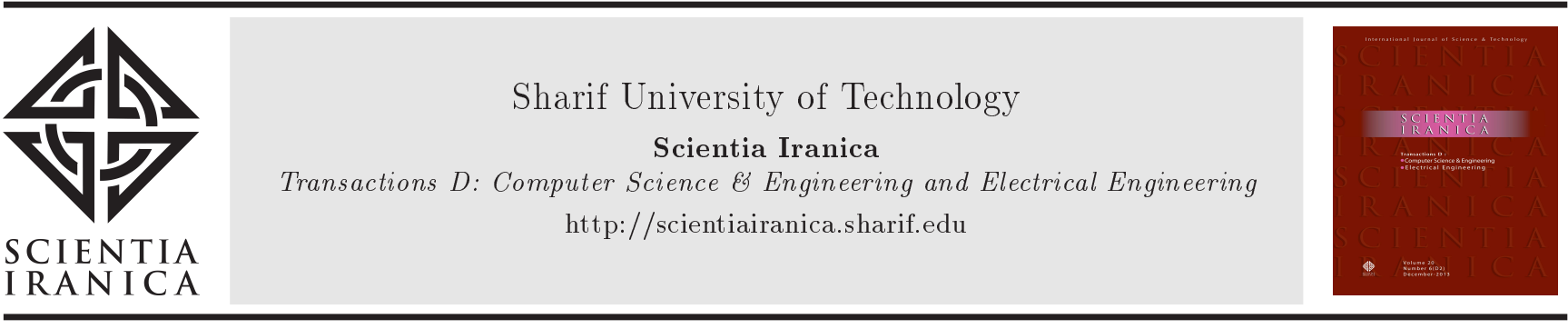

\title{
On the global practical stabilization of discrete-time switched affine systems: Application to switching power converters
}

\author{
M. Hejri* \\ Department of Electrical Engineering, Sahand University of Technology, Sahand New Town, Tabriz, P.O. Box 51335-1996, Iran.
}

Received 11 February 2020; received in revised form 24 August 2020; accepted 3 October 2020

\author{
KEYWORDS \\ Discrete-time switched \\ affine systems; \\ Stabilization; \\ Bilinear Matrix \\ Inequalities (BMIs); \\ DC-DC switching \\ power converters; \\ Practical stability.
}

\begin{abstract}
This paper presents new sufficient conditions as a set of Bilinear Matrix Inequalities (BMIs) for the globally practical stabilization of discrete-time switched affine systems. The main contribution of the current research involves proposing certain stability conditions based on a common quadratic Lyapunov function that can be used to stabilize the discrete-time switched affine systems around a desired equilibrium point for which it is not required to find any Schur stable convex combination of operating modes as a preprocessing stage. To this end, special algorithms are required for resolving an NP-hard problem. It is found that the existing two-stage stabilization methods based on a precalculation of a Schur stable convex combination of operating modes can be simplified into a single-stage method by which a high degree of applicability can be obtained. The proposed stability conditions are developed such that the size of the convergence ellipsoid is minimized. Moreover, it is not required for the equilibrium point around which the invariant set of attraction is constructed to be in a predetermined set of attainable equilibrium points. The satisfactory operation of the proposed stability conditions is illustrated with an academic example and the corresponding application to various DC-DC converters.
\end{abstract}

(C) 2021 Sharif University of Technology. All rights reserved.

\section{Introduction}

The general classes of hybrid dynamical systems involve both controlled and uncontrolled switching phenomena which make the analysis and synthesis of such systems quite complex and challenging [1-5]. In this regard, switched systems represent a special form of general hybrid dynamical systems that contain only controlled switching phenomena. Although switching patterns are restricted in this subclass of hybrid

*. Tel: +98 4133459363; Fax: +98 4123444322

E-mail address: hejri@sut.ac.ir

doi: $10.24200 /$ sci. 2020.55427 .4217 systems, many engineering and practical real-world systems can still fall into this class of hybrid systems of which one can mention power systems and power electronics, automotive control, aircraft and air-traffic control, and network control systems [6]. Although switched systems have been under scrutiny for long the instances of which can be found in a number of books [6-9] and survey papers [10-13], most of such works in this domain assume a common equilibrium point for all isolated subsystems. On the other hand, one of the most important subclasses of switched systems is the switched affine systems that are very common in practice, especially in power electronics area [14-19].

The main feature of switched affine systems is the existence of an affine term associated with each subsys- 
tem that keeps the equilibrium points of various affine subsystems from coinciding with each other. This implies that in a continuous-time domain, to maintain the state trajectories at a desired equilibrium point which is not necessarily aligned with the equilibrium point of each subsystem, the switching frequency approaches infinity $[20,21]$. It should be noted that such arbitrarily high-frequency switching may occur either during steady-state chattering when approaching the operating point or transient chattering due to the presence of sliding modes in the proposed controller [22]. However, such arbitrarily high-frequency switching employed to achieve asymptotic stability in the continuous time domain is not desirable from a practical point of view due to the possibility of equipment damage. To solve this problem in the continuous-time domain, a common practice is to consider an upper bound for the switching frequency and analyze the overall system as a sampled data switching system $[23,24]$. In some areas of relevant literature, this technique is introduced with the notion of a minimum dwell time by which the time interval between any two switching instants is guaranteed to not be smaller than a positive number called dwell time [25]. The limitation of switching frequency between various subsystems implies that the state trajectories converge to a region around the desired equilibrium instead of convergence to the equilibrium itself. This issue is reflected as the concept of practical stability in the literature [26-28].

In parallel with continuous time analysis and synthesis of switched affine systems, there are a few works dealing with the analysis of these systems in the discrete time setting $[20,21,29,30]$. Since any controller implementation is made via computers and discretetime samplers nowadays, discrete-time systems and their computer-based control are of particular importance. While stability conditions were formulated as a set of Bilinear Matrix Inequalities (BMIs) and Linear Matrix Inequalities (LMIs) in [20,21], a nonLMI based approach was established in $[29,30]$. Due to the capability and flexibility of LMIs and BMIs in formulation of various problems as well as their nice theoretical properties, they have received wide acceptance in academia and industry to formulate many optimization and control problems [31,32]. In this regard, the present paper provides the stability conditions based on a set of BMIs.

In [20], practical stability conditions were proposed for discrete-time switched affine systems via a common quadratic Lyapunov function. Compared to the present work, there are some limitations to the derived stability conditions in [20]. One limitation is that the invariant set of attraction must contain an equilibrium point that belongs to a predetermined set of attainable ones. This is a problem for the usability of the proposed conditions because these equilibrium points are generated via a Schur stable matrix calculated through the convex combination of each affine subsystem. On the other hand, checking the existence of a stable matrix as a convex combination of a family of matrices needs special algorithms and is an NP-hard problem [33]. Furthermore, in [20], the proposed stability conditions require a two-stage controller synthesis process: first, finding Lagrange multipliers $\lambda$ such that $A_{\lambda}$ is schur stable and second, utilizing these multipliers in a set of LMI or BMI conditions to find other unknown variables. Unfortunately, the implementation of the first stage involving the calculation of a Schur stable matrix with the respective weighting coefficients needs special algorithms and it is an NP-hard problem. On the other hand, in [20], no constructive approach was proposed to the calculation of such coefficients that weakened the applicability of the proposed LMI/BMI conditions. These limitations were addressed in [21] where the authors presented less conservative stability conditions via multiple Lyapunov functions. However, to achieve an invariant set of attraction, the authors used a rather complex two-stage algorithm using two different theorems with a large number of unknown variables. Of note, even in the continuous time domain, the existing works that do not need to precalculate a Hurwitz convex combination of affine subsystems utilize multiple Lyapunov functions [23,34-36]. However, compared to the single Lyapunov method, the stability conditions derived from multiple Lyapunov techniques represent more complicated matrix inequalities with many additional tuning parameters [23]. Therefore, in terms of complexity and usability, it is of particular importance to derive the stability conditions based on a single Lyapunov function without any need for determining a stable matrix as a convex combination of the affine subsystems in a preprocessing stage. This is the main mission and contribution of the present work.

In this respect, to the best of our knowledge, it is for the first time that the common quadratic Lyapunov function has been adopted in the context of the stabilization of discrete-time switched affine systems around a desired equilibrium point without belonging to any specific set and guaranteeing the invariance and attractive properties of a convergence set simultaneously. Moreover, in contrast to the existing two-stage stabilization methods, the stabilization process in the present work is made in a single stage without any need for pre-calculating a set of coefficients $\lambda$ such that $A_{\lambda}$ is schur stable. Indeed, the calculation of these variables is embedded inside the proposed BMI conditions. These advantages enlarge the application domain of the proposed stabilization technique.

Of note, although the proposed single-stage stability conditions are still BMI problems and computa- 
tionally demanding, there are several advantages and motivations for the proposed control design method.

First, let us assume that there is a tool that can find the global optimum solution to the BMIconstrained optimization problems. Such an assumption is reasonable at least for small-scale problems [37]. In the existing two-stage control design, first, we need to find suitable convex combination multipliers that yield Schur stability for the convex combination of the system matrices. Next, we need to solve a non-convex BMI problem to find the remaining set of unknown variables. However, this process should be repeated corresponding to all possible values of the convex combination multipliers to find the ultimate global optimum solution. One needs to implement the global optimization problem only once in the singlestage design and it is not required to run the repetitive global optimization programming. Therefore, finding the global optimal solution in the single-stage design is easier and more straightforward than that in the twostage one, at least from the implementation point of view.

Second, although BMI formulation for the control problems is not without its drawbacks and the major concern is related to the resulting non-convex optimization problem, the BMI formulation is still very important. The main advantage and attraction of the BMI formulation is related to its simplicity and generality. Moreover, it can easily accommodate many practical constraints during the controller synthesis for a physical plant. In addition, a wide range of the important robust control synthesis problems, even in the case of simple linear systems, are formulated in the BMI framework [38].

Finally, it should be noted that efficient BMI formulations play a key role between optimization techniques and solve many important control problems. One can always use the available nonlinear optimization techniques that at least ensure convergence to a local optimum solution. Even more, there are many works in the literature to find the global optimum point via global optimization methods such as various branch and bound techniques. A set of available methods can be found in [37] and references therein. Therefore, any progress in the classical nonlinear programming techniques from the computational point of view will lead to increased popularity and application domain of the BMI formulations.

According to the foregoing discussions, the main goal of this paper is to present new sufficient conditions based on a common quadratic Lyapunov function for the global practical stability of discrete-time switched affine systems by which the following properties are satisfied:

- Stabilization is made around any desired equilibrium point which does not belong to any predetermined set of attainable equilibrium points.

- In contrast to the existing two-stage stabilization methods, the proposed stabilization method is developed in a single stage in a sense that it is not required to compute any Schur stable matrix as a convex combination of a family of matrices in the pre-processing stage.

Notation. $\mathbb{R}, \mathbb{Z}_{\geq 0}$ are used to denote the set of real and nonnegative integer numbers, respectively. $\mathbb{R}^{n}$ and $\mathbb{R}^{m \times n}$ denote the set of real-valued $n$-dimensional column vectors and $m \times n$ matrices, respectively. We use $I_{n}$ and $0_{m \times n}$ to denote the $n \times n$ identity matrix and the $m \times n$ zero matrix, respectively. If $\lambda \in \mathbb{R}^{k}$ is a vector, then $\lambda_{i}$ is the $i^{\text {th }}$ element of $\lambda$. We use $\forall$ and $\exists$ to denote "for all" and "there exists", respectively. $\Rightarrow$ is used to denote logical implication. For matrix $M \in \mathbb{R}^{m \times n}$, $M^{T}$ denotes its transpose, and for a square matrix $M \in \mathbb{R}^{n \times n}, M^{-1}, \operatorname{tr}(M)$, and $\lambda_{i}(M)$ are inverse, trace and $i^{\text {th }}$ eigenvalue of $M$, respectively. Moreover $\lambda_{\max }(M)$ and $\lambda_{\min }(M)$ denote the largest and smallest eigenvalues of $M$, respectively. $M \prec 0$ and $M \preceq 0$ illustrate that $M$ is negative definite and negative semidefinite matrices, respectively. In symmetric matrices, * denotes each of their symmetric blocks. Assuming the set $\mathbb{K}=\{1, \ldots, N\}$ as a collection of the $N$ first positive integer numbers, the convex combination of matrices $\left\{M_{1}, \ldots, M_{N}\right\}$ is denoted by $M_{\lambda}=\sum_{i \in \mathbb{K}} \lambda_{i} M_{i}$ with $\lambda \in \Lambda$ where $\Lambda:=\left\{\lambda \in \mathbb{R}^{N} \mid \lambda_{i} \geq 0, \sum_{i \in \mathbb{K}} \lambda_{i}=1\right\}$ is the unitary simplex.

\section{Problem statement}

The discrete-time switched affine system is considered as follows:

$$
x(k+1)=A_{\sigma(x(k))} x(k)+b_{\sigma(x(k))}, x(0)=x_{0},
$$

where $k \in \mathbb{Z}_{\geq 0}$ is the discrete-time instant, $x(k) \in$ $\mathbb{R}^{n}$ is the state and $\sigma(x(k)): \mathbb{R}^{n} \rightarrow \mathbb{K}$ is a statedependent switching function that selects one of the $N$ available subsystems $\left(A_{i}, b_{i}\right), i \in \mathbb{K}$ at any instant of time $k \in \mathbb{Z}_{\geq 0}$. It is intended to design the statedependent switching function $\sigma(x(k))$ such that to impose asymptotic convergence of the state trajectories $x(k), k \in \mathbb{Z}_{\geq 0}$, to a neighborhood of the desired equilibrium point, for all initial conditions $x_{0} \in \mathbb{R}^{n}$. In general such equilibrium point does not coincide with any other of isolated subsystems, namely, $x_{e i}=\left(I_{n}-A_{i}\right)^{-1} b_{i}$. In some pieces of literature, in either continuous-time [14,39-41] or discrete-time [20] domain, it is assumed that this point belongs to a specific set of attainable equilibrium points. For instance, in [20], this set is defined by $X_{e}=\left\{x_{e} \in \mathbb{R}^{n} \mid x_{e}=\left(I_{n}-A_{\lambda}\right)^{-1} b_{\lambda}, \lambda \in S\right\}$ with $S \subseteq \Lambda$ such that $A_{\lambda}$ is Schur stable. In the present paper, in contrast to the existing literature based on the 
single Lyapunov function, such a limitation is relaxed and a different equilibrium point $x_{e} \notin X_{e}$ may be chosen. Furthermore, it is not required to compute any Schur stable matrix $A_{\lambda}$ by which $x_{e}$ is calculated. This is technically valuable because calculation of a stable matrix as a convex combination of operating modes needs special algorithms, thus making it an NP-hard problem [33]. Given an equilibrium point $x_{e}$, we can always reformulate the stabilization problem around the null equilibrium point by defining the error state vector $e(k)=x(k)-x_{e}, \forall k \in \mathbb{Z}_{>0}$ that follows the error dynamics below:

$$
e(k+1)=A_{\sigma(e(k))} e(k)+l_{\sigma(e(k))}, \quad e(0)=e_{0},
$$

with $\sigma(e(k))=\sigma\left(x(k)-x_{e}\right), l_{i}=\left(A_{i}-I_{n}\right) x_{e}+b_{i}, \forall i \in$ $\mathbb{K}$. We plan to design the switching function $\sigma(e(k))$ via a common quadratic Lyapunov function $v(e(k))=$ $e(k)^{T} \operatorname{Pe}(k), P=P^{T} \succ 0$ such that the ellipsoid $\mathcal{E}(P)=\left\{e(k) \in \mathbb{R}^{n} \mid e(k)^{T} P e(k) \leq 1\right\}$ with the center in the origin is an invariant set of attraction for the switched affine system (2) according to the following definition.

Definition 1. The ellipsoid $\mathcal{E}(P)=\left\{e \in \mathbb{R}^{n} \mid e^{T} P e \leq\right.$ $1\}$ is an invariant set of attraction in a given domain $D \subset \mathbb{R}^{n}$ for System (2) by the switching function $\sigma(e(k))$ if the following conditions are simultaneously satisfied:

(a) Invariant property: If $e(k) \in \mathcal{E}(P)$ then, e $(k+$ 1) $=A_{\sigma(e(k))} e(k)+l_{\sigma(e(k))} \in \mathcal{E}(P)$.

(b) Attractive property: If $e(0) \in D-\mathcal{E}(P)$, then there exist $T=T(e(0))>0$ and $\mathcal{E}(\hat{P}) \supset \mathcal{E}(P)$ with $\hat{P}=\hat{P}^{T} \succ 0$, such that $e(T) \in \mathcal{E}(P)$ and $e(k) \in \mathcal{E}(\hat{P}), \forall k \geq T$.

Based on Condition (a), the trajectories starting from $\mathcal{E}(P)$ are never escaped from it; therefore, due to the boundedness of $\mathcal{E}(P)$, they will remain around the null set point. If Condition (a) is satisfied it is called that the ellipsoid $\mathcal{E}(P)$ is an invariant for System (2) under switching function $\sigma(e(k))$. Furthermore, according to Condition (b), the trajectories starting outside $\mathcal{E}(P)$ evolve in time towards the point $e(k)=0_{n \times 1}$; however, they never reach it. According to this condition, the state trajectories eventually enter the ellipsoid $\mathcal{E}(P)$, but they may leave it. On the other hand, they will remain inside a larger ellipsoid $\mathcal{E}(\hat{P})$. In this case, the ellipsoid $\mathcal{E}(P)$ is considered attractive for System (2) under switching function $\sigma(e(k))$. Clearly, if Condition (a) is satisfied, then $\mathcal{E}(\hat{P})=\mathcal{E}(P)$ in Condition (b).

It should be noted that the notion of the set attraction in Definition 1 is a bit different from those available in the literature. In [20,42-44], a set $M$ is considered attraction set with respect to System (2) if any solution $e(k)$ of System (2) starting from $e(0) \notin M$ approaches the set $M$ when $k$ approaches infinity, i.e.:

$$
e(0) \notin M \Rightarrow \lim _{k \rightarrow \infty} \operatorname{dist}\{e(k), M\}=0,
$$

where $\operatorname{dist}\{e(k), M\}=\inf _{\rho \in M}|\rho-e(k)|$. According to $[26,27,29,45]$, the asymptotic practical stability of a set $M$ implies that for the state trajectories starting outside $M$, there is a finite time $T>0$ such that the trajectory $e(k)$ is ultimately inside $M$. However, unfortunately, the existing definition of the set attraction in Eq. (3) does not imply such property. In fact, it is only guaranteed that the distance between the state trajectory $e(k)$ and the set $M$ approaches zero, but it never enters $M$. On the other hand, Definition 1 falls in the category of practical stability by which it is meant that the trajectories either enter the ellipsoid $\mathcal{E}(P)$ or remain inside it. Definitions 2, 3, and 4 clarify these notions.

Definition 2. System (2) is locally practically stable with respect to an invariant set of attraction $\mathcal{E}(P)=$ $\left\{e \in \mathbb{R}^{n} \mid e^{T} P e \leq 1, P=P^{T} \succ 0\right\}$ in the domain $D$ under switching function $\sigma(e(k))$ if there exist sets $\mathcal{E}(P)$ and $D$ satisfying conditions of Definition 1 and $\mathcal{E}(P)=D$.

Of note, according to Definition 2, the concept of local practical stability is aligned with only invariance property of the ellipsoid $\mathcal{E}(P)$ introduced in Definition 1 for which only Condition (a) must be satisfied.

Definition 3. System (2) is practically stable in the large with respect to an invariant set of attraction $\mathcal{E}(P)$ in the domain $D$ under switching function $\sigma(e(k))$ if there exist sets $\mathcal{E}(P)$ and $D$ satisfying conditions of Definition 1 and $\mathcal{E}(P) \subset D$.

Definition 4. System (2) is practically stable in the whole or globally practically stable if it is practically stable in the large and that $D=\mathbb{R}^{n}$.

Definition 5. In Definitions 2-4, the set $D-\mathcal{E}(P)$ is called domain of attraction of System (2) under switching rule $\sigma(e(k))$.

Lemma 1 states that under what conditions System (2) is practically stable in the large in the sense of Definition 3 via Lyapunov functions.

Lemma 1. System (2) is practically stable in the large in a given domain $D \subset \mathbb{R}^{n}$ containing the origin in the sense of Definition 3 if there exist an ellipsoid $\mathcal{E}(P) \subset$ $D$ and a scalar function $v(e(k)): \mathbb{R}^{n} \rightarrow \mathbb{R}$ such that:

(a) If $e(k) \in \mathcal{E}(P)$, then $e(k+1)=A_{\sigma(e(k))} e(k)+$ $l_{\sigma(e(k))} \in \mathcal{E}(P)$; 
(b) If $e(k) \in D-\mathcal{E}(P)$, then $v(e(k+1))-v(e(k))=$ $\Delta v(e(k)) \leq-\gamma<0$ where $\gamma$ is a positive number;

(c) $v(e(k))$ is positive definite in $D-\mathcal{E}(P)$, i.e., $v(e(k))>0$ when $e(k) \in D-\mathcal{E}(P)$.

Proof. Condition (a) is the same as Condition (a) of Definition 1 and therefore, fulfills the invariant property of the ellipsoid $\mathcal{E}(P)$. To prove the attraction property of the set $\mathcal{E}(P)$, according to Condition (b) of Definition 1 it is necessary to demonstrate that upon starting from any initial state $e(0) \in D-\mathcal{E}(P)$, there exists a finite time $T=T(e(0))>0$ such that for $k \geq T$, the state $e(k)$ enters the ellipsoid $\mathcal{E}(P)$, i.e., $\exists T>0$ such that $e(k) \in \mathcal{E}(P)$ for $k \geq T$. This can be demonstrated by contradiction as follows. Assume that the state trajectory $e(k)$ never intersects with $\mathcal{E}(P)$. Then, at any instant $k$ outside $\mathcal{E}(P)$ and according to Condition (b), we have $v(e(k+1))-v(e(k)) \leq-\gamma<0$. In view of this, one can write the following:

$$
\begin{aligned}
v(e(k)) & =v(e(0))+\sum_{n=0}^{k-1}(v(e(n+1)-v(e(n))) \\
& \leq v(e(0))-k \gamma .
\end{aligned}
$$

The right-hand side of Eq. (4) will be eventually negative when $k$ takes large values. This leads to contradiction against Condition (c) where it is assumed that $v(e(k))$ is positive definite on $D-\mathcal{E}(P)$. As a result, the state trajectory $e(k)$ will eventually enter the ellipsoid $\mathcal{E}(P)$ at finite time $T$ and remains within it $(\forall k \geq T)$ due to the Condition (a). Therefore, $\mathcal{E}(P)$ is an invariant set of attraction according to Definition 1 and as a result, according to Definition 3, System (2) is practically stable in the large in the domain $D$ and under switching function $\sigma(e(k))$. Thus, the proof is completed.

Remark 1. In Lemma 1, Conditions (b) and (c) imply Condition (b) of Definition 1. In the proof of Lemma 1, it was verified that any trajectory starting from outside ellipsoid $\mathcal{E}(P)$ would eventually enter it after finite steps $T$. Now, if the invariance condition is not satisfied, then the trajectory may leave $\mathcal{E}(P)$. However, it is guaranteed that there is a larger ellipsoid $\mathcal{E}(\hat{P}) \supset \mathcal{E}(P)$ with $\hat{P}=\hat{P}^{T} \succ 0$, such that $e(k) \in \mathcal{E}(\hat{P})$, $\forall k \geq T$. This can be shown by contradiction. Let us assume that there is no ellipsoid and the trajectories will be unbounded after reaching inside $\mathcal{E}(P)$. Then, there would exist $T_{2}>T_{1}>T$ such that $e\left(T_{2}\right) \notin \mathcal{E}(P)$, $e\left(T_{1}\right) \notin \mathcal{E}(P)$, and $v\left(e\left(T_{2}\right)\right)>v\left(e\left(T_{1}\right)\right)$. However, this cannot occur since $v(e(k))$ is strictly decreasing outside ellipsoid $\mathcal{E}(P)$.

Remark 2. Definitions 1-5, Lemma 1 and its proof can be applied to the autonomous nonlinear switched systems without a common equilibrium point where the functions $f_{i}(e(k)), i \in \mathbb{K}$ in $e(k+1)=f_{i}(e(k))$ do not necessarily need to be in the affine form as in System (2). In this context, one should replace Condition (b) in Definition 1 and Lemma 1 by the following one: (b) If $e(k) \in \mathcal{E}(P)$, then $e(k+1)=$ $f_{\sigma(e(k))}(e(k)) \in \mathcal{E}(P)$.

Remark 3. In Lemma 1, if $D=\mathbb{R}^{n}$, then System (2) is globally practically stable according to Definition 4 .

\section{Stability analysis}

In this section, the main result of this paper is presented. As discussed earlier, we are planning to design a switching rule $\sigma(e(k)), k \in \mathbb{Z}_{\geq 0}$ for System (2) that drives the state trajectories towards the ellipsoid $\mathcal{E}(P)=\left\{e \in \mathbb{R}^{n} \mid e^{T} P e \leq 1\right\}$ with a center in the origin. In this paper, a min-type state feedback switching function is used as follows:

$$
\sigma(e(k))=\arg \min _{i \in \mathbb{K}} v\left(A_{i} e(k)+l_{i}\right)
$$

Lemmas 2 and 3 are frequently used in proving Theorem 1.

Lemma 2. For a set of functions $f_{i}: D \rightarrow \mathbb{R}, D \subseteq$ $\mathbb{R}^{n}, i \in \mathbb{K}$ as $f_{1}(x), \ldots, f_{l}(x), \ldots, f_{N}(x)$, the following statements are equivalent.

i) $\forall x \in D, \exists l \in \mathbb{K}$, such that $f_{l}(x)<0$;

ii) $\forall x \in D, \exists\left(\lambda_{1} \geq 0, \ldots, \lambda_{l} \geq 0, \ldots, \lambda_{N} \geq 0\right)$ such that $\sum_{i \in \mathbb{K}} \lambda_{i} f_{i}(x)<0$, and $\sum_{i \in \mathbb{K}} \lambda_{i}>0$.

Proof. $i) \Rightarrow i i)$

Let us assume that $\forall x \in D \subseteq \mathbb{R}^{n}$, at least one of the functions, say $f_{l}(x), l \in \mathbb{K}$ satisfies the inequality $f_{l}(x)<0$. Now, by choosing the set of parameters $\lambda_{l}>$ $0, \lambda_{i}=0, i \neq l, i \in \mathbb{K}$, one can conclude $\sum_{i \in \mathbb{K}} \lambda_{i} f_{i}(x)=$ $\lambda_{l} f_{l}(x)<0$ and $\sum_{i \in \mathbb{K}} \lambda_{i}=\lambda_{l}>0$.

ii) $\Rightarrow i$ )

This can be shown by contradiction: Let us assume $\exists x \in D \subseteq \mathbb{R}^{n}$ such that all $f_{l}(x), l \in \mathbb{K}$ fulfill $f_{l}(x) \geq 0$. By multiplication of these inequalities to $\lambda_{l} \geq 0$ such that $\sum_{i \in \mathbb{K}} \lambda_{i}>0$, and summing up all the terms, one can reach $\sum_{l \in \mathbb{K}} \lambda_{l} f_{l}(x) \geq 0$. However, this contradicts the fact that $\forall x \in \mathbb{R}^{n}$ there exists a set of nonnegative parameters $\lambda_{l} \geq 0, l \in \mathbb{K}$ such that $\sum_{i \in \mathbb{K}} \lambda_{i} f_{i}(x)<0$. Lemma 3 is a nonstrict form of Lemma 2 .

Lemma 3. For a set of functions $f_{i}: \mathbb{R}^{n} \rightarrow \mathbb{R}, i \in$ $\mathbb{K}$ as $f_{1}(x), \ldots, f_{l}(x), \ldots, f_{N}(x)$, the following statements are equivalent:

i) $\forall x \in \mathbb{R}^{n}, \exists l \in \mathbb{K}$ such that $f_{l}(x) \geq 0$; 
ii) $\forall x \in \mathbb{R}^{n}, \exists\left(\lambda_{1} \geq 0, \ldots, \lambda_{l} \geq 0, \ldots, \lambda_{N} \geq 0\right)$ such that $\sum_{i \in \mathbb{K}} \lambda_{i} f_{i}(x) \geq 0$, and $\sum_{i \in \mathbb{K}} \lambda_{i}>0$.

Proof. The proof is similar to that of Lemma 2 and is omitted for the sake of brevity. $\square$

Theorem 1 provides sufficient conditions for which the switched affine system (2) under switching function (5) is globally practically stable in the sense of Definition 1.

Theorem 1. If there exist matrix $P=P^{T} \succ 0$ and nonnegative numbers $\lambda_{1 i} \geq 0, \lambda_{2 i} \geq 0, i \in \mathbb{K}, \tau_{1} \geq 0$, $\tau_{2} \geq 0$ satisfying the system of inequalities:

$$
\begin{aligned}
& {\left[\begin{array}{cc}
M_{1}-\tau_{1} P & * \\
M_{2} & M_{3}+\tau_{1}-\sum_{i \in \mathbb{K}} \lambda_{1 i}
\end{array}\right] \preccurlyeq 0,} \\
& {\left[\begin{array}{cc}
\tau_{2} P+\tilde{M}_{1}-\sum_{i \in \mathbb{K}} \lambda_{2 i} P & \tilde{M}_{3}-\tau_{2}
\end{array}\right] \prec 0,} \\
& \sum_{i \in \mathbb{K}} \lambda_{1 i}>0, \\
& \sum_{i \in \mathbb{K}} \lambda_{2 i}>0,
\end{aligned}
$$

where:

$$
\begin{aligned}
& M_{1}=\sum_{i \in \mathbb{K}} \lambda_{1 i} A_{i}^{T} P A_{i}, \\
& M_{2}=\sum_{i \in \mathbb{K}} \lambda_{1 i} l_{i}^{T} P A_{i}, \\
& M_{3}=\sum_{i \in \mathbb{K}} \lambda_{1 i} l_{i}^{T} P l_{i}, \\
& \tilde{M}_{1}=\sum_{i \in \mathbb{K}} \lambda_{2 i} A_{i}^{T} P A_{i}, \\
& \tilde{M}_{2}=\sum_{i \in \mathbb{K}} \lambda_{2 i} l_{i}^{T} P A_{i}, \\
& \tilde{M}_{3}=\sum_{i \in \mathbb{K}} \lambda_{2 i} l_{i}^{T} P l_{i},
\end{aligned}
$$

then the switching strategy in $E q$. (5) ensures that the ellipsoid $\mathcal{E}(P)=\left\{e \in \mathbb{R}^{n} \mid e^{T} P e \leq 1\right\}$ with $k \in \mathbb{Z}_{\geq 0}$ is invariant set of attraction for discrete-time switched affine system in Eq. (2).

Proof. It is shown that Relations (6) and (8) imply Condition (a) of Lemma 1, while Relations (7) and (9) fulfill Condition (b) via $v(e(k))=e(k)^{T} P e(k)$ satisfying Condition (c) of Lemma 1. Pre-multiplying Relation (6) by $\left[e(k)^{T} 1\right]$ and post-multiplying it by $\left[e(k)^{T} 1\right]^{T}$, one can reach:

$$
\begin{gathered}
{\left[\begin{array}{c}
e(k) \\
1
\end{array}\right]^{T}\left[\begin{array}{cc}
M_{1}-\tau_{1} P & * \\
M_{2} & M_{3}+\tau_{1}-\sum_{i \in \mathbb{K}} \lambda_{1 i}
\end{array}\right]\left[\begin{array}{c}
e(k) \\
1
\end{array}\right]} \\
\leq 0, \quad \forall e(k) \in \mathbb{R}^{n} .
\end{gathered}
$$

Relation (16) can be rewritten as Relation (17):

$$
\begin{array}{r}
{\left[\begin{array}{c}
e(k)]^{T} \\
1
\end{array}\right]^{T}\left[\begin{array}{cc}
M_{1} & M_{3}-\sum_{i \in \mathbb{K}}^{*} \lambda_{1 i}
\end{array}\right]\left[\begin{array}{c}
e(k) \\
1
\end{array}\right]-\tau_{1}\left[\begin{array}{c}
e(k) \\
1
\end{array}\right]^{T}} \\
{\left[\begin{array}{cc}
P & * \\
0_{1 \times n} & -1
\end{array}\right]\left[\begin{array}{c}
e(k) \\
1
\end{array}\right] \leq 0, \quad \forall e(k) \in \mathbb{R}^{n} .}
\end{array}
$$

Using S-procedure [31,37], Relation (17) implies Relation (18).

$$
\begin{array}{r}
{\left[\begin{array}{c}
e(k) \\
1
\end{array}\right]^{T}\left[\begin{array}{cc}
P & * \\
0_{1 \times n} & -1
\end{array}\right]\left[\begin{array}{c}
e(k) \\
1
\end{array}\right] \leq 0 \Rightarrow\left[\begin{array}{c}
e(k) \\
1
\end{array}\right]^{T}} \\
{\left[\begin{array}{ll}
M_{1} & M_{3}-\sum_{i \in \mathbb{K}}^{*} \lambda_{1 i}
\end{array}\right]\left[\begin{array}{c}
e(k) \\
1
\end{array}\right] \leq 0 .}
\end{array}
$$

By substituting $M_{1}, M_{2}$, and $M_{3}$ from Eqs. (10)-(12) into Relation (18), one can write:

$$
\begin{array}{rl}
e(k)^{T} & P e(k) \leq 1 \Rightarrow \sum_{i \in \mathbb{K}} \lambda_{1 i}\left(e(k)^{T} A_{i}^{T} P A_{i} e(k)\right. \\
& \left.+l_{i}^{T} P A_{i} e(k)+e(k)^{T} A_{i}^{T} P l_{i}+l_{i}^{T} P l_{i}-1\right) \leq 0 .
\end{array}
$$

According to Relation (8), since $\sum_{i \in \mathbb{K}} \lambda_{1 i}>0, \lambda_{1 i} \geq$ $0, i \in \mathbb{K}$, Lemma 3 implies that Relation (19) can be rewritten as:

$$
\begin{aligned}
& e(k)^{T} P e(k) \leq 1 \Rightarrow \exists i \in \mathbb{K} \text { such that } \\
& e(k)^{T} A_{i}^{T} P A_{i} e(k)+l_{i}^{T} P A_{i} e(k)+e(k)^{T} A_{i}^{T} P l_{i} \\
& \quad+l_{i}^{T} P l_{i}-1 \leq 0 .
\end{aligned}
$$

Relation (20) implies that for each $e(k)$ satisfying $e(k)^{T} P e(k) \leq 1$, there exists an index $i$ such that $e(k)^{T} A_{i}^{T} P A_{i} e(k)+l_{i}^{T} P A_{i} e(k)+e(k)^{T} A_{i}^{T} P l_{i}+l_{i}^{T} P l_{i}-$ $1 \leq 0$. This is because the switching rule is not constant inside the set $\mathcal{E}(P)=\left\{e(k) \in \mathbb{R}^{n} \mid e(k)^{T} P e(k) \leq 1\right\}$. This statement also holds for the next similar logical expressions. After performing some algebra on the right-hand side of Relation (20), one can reach:

$$
\begin{gathered}
e(k)^{T} P e(k) \leq 1 \Rightarrow \exists i \in \mathbb{K} \text { such that } \\
\left(A_{i} e(k)+l_{i}\right)^{T} P\left(A_{i} e(k)+l_{i}\right) \leq 1,
\end{gathered}
$$

which can be rewritten as:

$$
\begin{aligned}
& e(k)^{T} P e(k) \leq 1 \Rightarrow \exists i \in \mathbb{K} \text { such that } \\
& v\left(A_{i} e(k)+l_{i}\right) \leq 1 .
\end{aligned}
$$

Based on the switching rule in Eq. (5), since $v\left(A_{\sigma} e(k)+\right.$ $\left.l_{\sigma}\right) \leq v\left(A_{i} e(k)+l_{i}\right), \forall i \in \mathbb{K}$, from Relation (22), one can conclude that: 


$$
e(k)^{T} P e(k) \leq 1 \Rightarrow v\left(A_{\sigma(e(k))} e(k)+l_{\sigma(e(k))}\right) \leq 1 .
$$

Since, according to System $(2), e(k+1)=A_{\sigma(e(k))} e(k)$ $+l_{\sigma(e(k))}$, Relation (23) can be rewritten as follows:

$$
e(k)^{T} \operatorname{Pe}(k) \leq 1 \Rightarrow e(k+1)^{T} \operatorname{Pe}(k+1) \leq 1 .
$$

Relation (24) states that whenever $e(k) \in \mathcal{E}(P), e(k+$ 1) $\in \mathcal{E}(P)$ as well. Thus, according to Condition (a) of Lemma 1, Relation (24) implies that the ellipsoid $\mathcal{E}(P)=\left\{e \in \mathbb{R}^{n} \mid e^{T} P e \leq 1\right\}$ is an invariant set for the switched affine system (2) under switching rule (Eq. (5)). In the sequel, we plan to prove the attraction of the set $\mathcal{E}(P)$ via Relations $(7)$ and $(8)$ and $v(e(k))=$ $e(k)^{T} P e(k)$. Pre-multiplying Relation $(7)$ by $\left[e(k)^{T} 1\right]$ and post-multiplying it by $\left[e(k)^{T} 1\right]^{T}$, one can obtain:

$$
\begin{gathered}
{\left[\begin{array}{c}
e(k) \\
1
\end{array}\right]^{T}\left[\begin{array}{cc}
\tau_{2} P+\tilde{M}_{1}-\sum_{i \in \mathbb{K}} \lambda_{2 i} P & * \\
\tilde{M}_{2} & \tilde{M}_{3}-\tau_{2}
\end{array}\right]} \\
{\left[\begin{array}{c}
e(k) \\
1
\end{array}\right]<0, \quad \forall e(k) \in \mathbb{R}^{n} .}
\end{gathered}
$$

Relation (25) can be rewritten into Relation (26):

$$
\begin{gathered}
{\left[\begin{array}{c}
e(k) \\
1
\end{array}\right]^{T}\left[\begin{array}{cc}
\tilde{M}_{1}-\sum_{i \in \mathbb{K}} \lambda_{2 i} P & * \\
\tilde{M}_{2} & \tilde{M}_{3}
\end{array}\right]\left[\begin{array}{c}
e(k) \\
1
\end{array}\right]-\tau_{2}} \\
{\left[\begin{array}{c}
e(k) \\
1
\end{array}\right]^{T}\left[\begin{array}{cc}
-P & * \\
0_{1 \times n} & 1
\end{array}\right]\left[\begin{array}{c}
e(k) \\
1
\end{array}\right]<0,}
\end{gathered}
$$

$\forall e(k) \in \mathbb{R}^{n}$.

Using S-procedure, Relation (26) is substituted into Relation (27):

$$
\begin{aligned}
& {\left[\begin{array}{c}
e(k) \\
1
\end{array}\right]^{T}\left[\begin{array}{cc}
-P & * \\
0_{1 \times n} & 1
\end{array}\right]\left[\begin{array}{c}
e(k) \\
1
\end{array}\right]<0 \Rightarrow} \\
& \quad\left[\begin{array}{c}
e(k) \\
1
\end{array}\right]^{T}\left[\begin{array}{cc}
\tilde{M}_{1}-\sum_{i \in \mathbb{K}} \lambda_{2 i} P & * \\
\tilde{M}_{2} & \tilde{M}_{3}
\end{array}\right]\left[\begin{array}{c}
e(k) \\
1
\end{array}\right] \\
& \quad<0 .
\end{aligned}
$$

By substituting $\tilde{M}_{1}, \tilde{M}_{2}$, and $\tilde{M}_{3}$ from Eqs. (13)-(15) into Relation (27) and after doing some algebra, one can reach:

$$
\begin{array}{r}
e(k)^{T} P e(k)>1 \Rightarrow \sum_{i \in \mathbb{K}} \lambda_{2 i}\left[\left(A_{i} e(k)+l_{i}\right)^{T}\right. \\
\left.P\left(A_{i} e(k)+l_{i}\right)-e(k)^{T} \operatorname{Pe}(k)\right]<0 .
\end{array}
$$

According to Relation (9), since $\sum_{i \in \mathbb{K}} \lambda_{2 i}>0, \lambda_{2 i} \geq$ $0, i \in \mathbb{K}$, Lemma 2 implies that Relation (28) can be rewritten as follows:

$$
\begin{aligned}
& e(k)^{T} \operatorname{Pe}(k)>1 \Rightarrow \exists i \in \mathbb{K} \text { such that } \\
& \left(A_{i} e(k)+l_{i}\right)^{T} P\left(A_{i} e(k)+l_{i}\right)-e(k)^{T} P e(k)<0 .
\end{aligned}
$$

Relation (29) implies that for each $e(k)$ to satisfy $e(k)^{T} \operatorname{Pe}(k)>1$, there exists an index $i$ such that $\left(A_{i} e(k)+l_{i}\right)^{T} P\left(A_{i} e(k)+l_{i}\right)-e(k)^{T} P e(k)<0$. This is because outside the set $\mathcal{E}(P)=\{e(k) \in$ $\left.\mathbb{R}^{n} \mid e(k)^{T} \operatorname{Pe}(k) \leq 1\right\}$, the switching function is not constant. According to the switching rule (5), we have:

$$
\begin{gathered}
\left(A_{\sigma(e(k))} e(k)+l_{\sigma(e(k))}\right)^{T} P\left(A_{\sigma(e(k))} e(k)+l_{\sigma(e(k))}\right) \\
\leq\left(A_{i} e(k)+l_{i}\right)^{T} P\left(A_{i} e(k)+l_{i}\right) .
\end{gathered}
$$

From Relations (29) and (30), one can reach:

$$
\begin{array}{rl}
e(k)^{T} & P e(k)>1 \Rightarrow\left(A_{\sigma(e(k))} e(k)+l_{\sigma(e(k))}\right)^{T} \\
& P\left(A_{\sigma(e(k))} e(k)+l_{\sigma(e(k))}\right)-e(k)^{T} \operatorname{Pe}(k) \\
\leq & \left(A_{i} e(k)+l_{i}\right)^{T} P\left(A_{i} e(k)+l_{i}\right) \\
- & e(k)^{T} \operatorname{Pe}(k)<0,
\end{array}
$$

or equivalently:

$$
\begin{array}{rl}
e(k)^{T} & P e(k)>1 \Rightarrow v\left(A_{\sigma(e(k))} e+l_{\sigma(e(k))}\right)-v(e(k)) \\
& =v(e(k+1))-v(e(k))=\Delta v(e(k)) \\
& \leq\left(A_{i} e(k)+l_{i}\right)^{T} P\left(A_{i} e(k)+l_{i}\right) \\
& -e(k)^{T} P e(k)<0
\end{array}
$$

which implies some part of Condition (b) in Lemma 1. According to item (b) of Lemma 1, we still need to find a positive number $\gamma$ such that $\Delta v(e(k)) \leq-\gamma<0$ when $e(k) \in D-\mathcal{E}(P)$. In this regard, we define the following:

$$
\begin{gathered}
\phi_{j}(e(k))=e(k)^{T} \operatorname{Pe}(k)-\left(A_{j} e(k)+l_{j}\right)^{T} \\
P\left(A_{j} e(k)+l_{j}\right), j \in S, \\
S=\left\{j \in \mathbb{K} \mid \Delta v(e(k)) \leq-\phi_{j}(e(k))<0\right\} .
\end{gathered}
$$

According to Relation (32), $|S| \geq 1$ and $\phi_{j}(e(k))>0$ when $e(k) \notin \mathcal{E}(P)$. Now, we define the function $\phi(s)$ as follows:

$$
\begin{aligned}
\phi(s)= & \inf _{s \leq\|e(k)\|} \min _{j \in S} \phi_{j}(e(k)), \\
& s \geq 0 \\
& e(k) \notin \mathcal{E}(P)
\end{aligned}
$$

The function $\phi(\|e(k)\|)$ is nondecreasing and positive definite when $e(k) \notin \mathcal{E}(P)$. Moreover, according to Relations (35) and (32), one can write:

$$
\Delta v(e(k)) \leq-\phi_{j}(e(k)) \leq-\phi(\|e(k)\|)<0, j \in S
$$


According to Relation (35), since $e(k) \notin \mathcal{E}(P)$, $e(k)^{T} P e(k)>1$. For a symmetric matrix $P=P^{T} \in$ $\mathbb{R}^{n \times n}$, we have $e(k)^{T} P e(k) \leq \lambda_{\max }(P)\|e(k)\|^{2}$ which in combination with $e(k)^{T} P e(k)>1$ yields $\|e(k)\| \geq$ $\frac{1}{\sqrt{\lambda_{\max }(P)}}$. As a result, due to the nondecreasing nature of function $\phi(s)$ in Relation (35), one can reach:

$$
\phi(\|e(k)\|) \geq \phi\left(\frac{1}{\sqrt{\lambda_{\max }(P)}}\right)=\gamma,
$$

which together with Relation (36) leads to the following:

$$
\Delta v(e(k)) \leq-\phi_{j}(e(k)) \leq-\phi(\|e(k)\|) \leq-\gamma<0,
$$

$$
j \in S,
$$

which meets Condition (b) in Lemma 1 . Since $P=$ $P^{T} \succ 0, v(e(k))=e(k)^{T} P e(k)$ is positive definite in the whole state space unless $e(k)=0_{n \times 1}$ which satisfies Condition (c) of Lemma 1. Therefore, all conditions of Lemma 1 are satisfied. Since during proof of Theorem 1 no restriction is imposed on the selection of $e(k)$, i.e., $e(k) \in \mathbb{R}^{n}$, as a result $D=\mathbb{R}^{n}$ and according to Remark 3 the switched affine system (2) is globally practically stable under switching rule (5) and the proof is concluded.

Remark 4. It should be noted that the inequalities appearing in Relations (6) and (7) are not convex with respect to $\lambda_{i}, P, \tau_{1}$, and $\tau_{2}$ and they are a set of BMIs. Nevertheless, the LMI and BMI conditions proposed in [20] are developed in a double-stage process during which at first it is necessary to calculate a set of coefficients $\lambda_{i} \in \Lambda$ such that $A_{\lambda}=\sum_{i \in \mathbb{K}} \lambda_{i} A_{i}$ is a Schur stable matrix. Next, upon utilizing these precalculated coefficients within a set of LMI and BMI conditions, other unknown variables are computed. As already discussed, unfortunately, the calculation of such a stable matrix with the respective weighting coefficients needs special algorithms, thus making it an NP-hard problem. However, as it can be observed, such a requirement does not appear in the conditions of Theorem 1 and stabilization process is made in a single stage without need for the precalculation of a set of coefficients $\lambda_{2 i} \in \Lambda$ such that $A_{\lambda}=\sum_{i \in \mathbb{K}} \lambda_{2 i} A_{i}$ is a Schur stable matrix. Obviously, this does not mean that such a stable convex combination does not exist. Corollary 1 highlights this issue.

Corollary 1. Condition (7) in Theorem 1 implies the existence of a Schur stable convex combination of subsystem matrices $A_{\lambda}=\sum_{i \in \mathbb{K}} \frac{\lambda_{2 i}}{\sum_{i \in \mathbb{K}} \lambda_{2 i}} A_{i}$.

Proof. According to the Schur complements [31] for strict inequalities, Condition (7) takes Relation (38) as a necessary condition.

$$
\sum_{i \in \mathbb{K}} \lambda_{2 i} A_{i}^{T} P A_{i}-\sum_{i \in \mathbb{K}} \lambda_{2 i} P \prec 0 .
$$

In [46], it was shown that:

$$
\left[\begin{array}{cc}
A_{i}^{T} P A_{i} & A_{i}^{T} P \\
P A_{i} & P
\end{array}\right] \succeq 0 .
$$

By multiplying Inequality (39) by $\lambda_{2 i} \geq 0$, and summing up for all $i \in \mathbb{K}$, one can reach:

$$
\left[\begin{array}{cc}
\sum_{i \in \mathbb{K}} \lambda_{2 i} A_{i}^{T} P A_{i} & \sum_{i \in \mathbb{K}} \lambda_{2 i} A_{i}^{T} P \\
\sum_{i \in \mathbb{K}} \lambda_{2 i} P A_{i} & P \sum_{i \in \mathbb{K}} \lambda_{2 i}
\end{array}\right] \succeq 0 .
$$

Performing the Schur complements for non-strict inequalities [31] with respect to the term in the second row and second column and noting that $\sum_{i \in \mathbb{K}} \lambda_{2 i}>0$, one can reach:

$$
\begin{aligned}
\sum_{i \in \mathbb{K}} \lambda_{2 i} A_{i}^{T} P A_{i} & -\frac{\left(\sum_{i \in \mathbb{K}} \lambda_{2 i} A_{i}^{T}\right) P P^{-1} P\left(\sum_{i \in \mathbb{K}} \lambda_{2 i} A_{i}\right)}{\sum_{i \in \mathbb{K}} \lambda_{2 i}} \\
& \succeq 0 .
\end{aligned}
$$

Now, from Relations (38) and (41), one can infer:

$$
\frac{\left(\sum_{i \in \mathbb{K}} \lambda_{2 i} A_{i}^{T}\right) P\left(\sum_{i \in \mathbb{K}} \lambda_{2 i} A_{i}\right)}{\left(\sum_{i \in \mathbb{K}} \lambda_{2 i}\right)^{2}} \prec P,
$$

Relation (42) can be rewritten as:

$$
\left(\sum_{i \in \mathbb{K}} \frac{\lambda_{2 i}}{\sum_{i \in \mathbb{K}} \lambda_{2 i}} A_{i}^{T}\right) P\left(\sum_{i \in \mathbb{K}} \frac{\lambda_{2 i}}{\sum_{i \in \mathbb{K}} \lambda_{2 i}} A_{i}\right) \prec P .
$$

From Relation (43), it can be concluded $A_{\lambda}=$ $\sum_{i \in \mathbb{K}} \frac{\lambda_{2 i}}{\sum_{i \in \mathbb{K}} \lambda_{2 i}} A_{i}$ is Schur stable where $\frac{\lambda_{2 i}}{\sum_{i \in \mathbb{K}} \lambda_{2 i}} \in \Lambda$. In other words, in the proposed stability conditions, the Schur stability of matrix $A_{\lambda}$ is a necessary condition for attraction condition (7) of Theorem $1 . \square$

\section{Minimization of the invariant set of attraction}

There are two main objective functions for the size minimization of ellipsoid $\mathcal{E}(P)=\left\{e \in \mathbb{R}^{n} \mid e^{T} P e \leq\right.$ $1\}, k \in \mathbb{Z}_{\geq 0}$ under the proposed BMI constraints in Theorem 1. One technique is to minimize the ellipsoid volume through the minimization of $\operatorname{det}\left(P^{-1}\right)$ $[19,20,31]$. In [42], it is discussed since:

$$
\operatorname{det}\left(P^{-1}\right)=\prod_{i=1}^{n} \lambda_{i}\left(P^{-1}\right)=\prod_{i=1}^{n} \rho_{i}^{2}\left(P^{-1}\right),
$$

where $\rho_{i}\left(P^{-1}\right)$ is the distance from the center to each semiaxis of the ellipsoid $\mathcal{E}(P)$, the product $\prod_{i=1}^{n} \rho_{i}\left(P^{-1}\right)$ may take a very large value of one semiaxis, say $\rho_{j}\left(P^{-1}\right)$, while all others may take very small values. As a result, minimum volume criterion 
through the minimization of $\operatorname{det}\left(P^{-1}\right)$ may not ensure good performance in all directions.

Another approach is to use the trace of the matrix $P^{-1}$ that defines the sum of the squares of the ellipsoid $\mathcal{E}(P)$ semiaxes as follows [42]:

$$
\min _{P \succ 0, \lambda_{1 i} \geq 0, \lambda_{2 i} \geq 0, \tau_{1} \geq 0, \tau_{2} \geq 0} \operatorname{tr}\left(P^{-1}\right)
$$

subject to Relations $(6)-(9)$.

Since the tool YALMIP/MATLAB [47] was not able to handle the objective function $\operatorname{tr}\left(P^{-1}\right)$, we modified it such that the software limitation could be relaxed and the main property of the size minimization be preserved. Note that since $P \succ 0$, we have:

$$
\begin{aligned}
\operatorname{tr}\left(P^{-1}\right) & =\sum_{i=1}^{i=n} \lambda_{i}\left(P^{-1}\right) \leq n \max _{i=1, \ldots, n} \lambda_{i}\left(P^{-1}\right) \\
& =n \lambda_{\max }\left(P^{-1}\right) .
\end{aligned}
$$

Therefore, on the one hand, the minimization of the maximum eigenvalue of $P^{-1}$ guarantees the minimization of the corresponding maximal semiaxis $\rho_{\max }\left(P^{-1}\right)=\sqrt{\lambda_{\max }\left(P^{-1}\right)}$ and minimizes an upperbound for $\operatorname{tr}\left(P^{-1}\right)$. On the other hand, the minimization of the $\lambda_{\max }\left(P^{-1}\right)$ is equivalent to the following minimization problem [32]:

$$
\begin{array}{cc}
\min & t \\
\text { subject to } & I_{n}-t P \preceq 0
\end{array}
$$

Accordingly, Relation (45) is replaced by the following optimization problem:

$$
\min _{P \succ 0, \lambda_{1 i} \geq 0, \lambda_{2 i} \geq 0, i \in \mathbb{K}, \tau_{1} \geq 0, \tau_{2} \geq 0} t
$$

subject to Relations $(6)-(9)$

$$
I_{n}-t P \preceq 0 .
$$

In this paper, the optimization problem in Relation (48) is solved via the BMI solver PENBMI [48] interfaced by YALMIP [47]. In the course of numerical experiments in the next section, we realize that the optimization problem in Relation (48) is still very nonlinear and non-convex in some cases to achieve suitable and acceptable results from the PENBMI package. It is noted that an option to limit the search space, is to consider upper bounds to the sum of the lambda's as $\sum_{i \in \mathbb{K}} \lambda_{1 i} \leq h_{1}, h_{1}>0, \sum_{i \in \mathbb{K}} \lambda_{2 i} \leq h_{2}, h_{2}>0$; however, unfortunately, the PENBMI solver did not converge to an acceptable solution. In this regard, stronger equality constraints $\sum_{i \in \mathbb{K}} \lambda_{1 i}=h_{1}, h_{1}>0$, $\sum_{i \in \mathbb{K}} \lambda_{2 i}=h_{2}, h_{2}>0$ are considered in Relation (48) to limit the search space and achieve desirable results from the solver, but at the expense of conservatism. It should be noted that the values of $h_{1}$ and $h_{2}$ do not have any effect on the values of the matrix $P$ and parameter $t$ in the optimization problem (48); as a result, changing their values does not change the size of the invariant set of attraction. According to the numerical observations with a change in $h_{1}$ and $h_{2}$, the values of variables $\tau_{1}, \lambda_{1 i}, \tau_{2}$, and $\lambda_{2 i}$ are scaled proportional to the values of $h_{1}$ and $h_{2}$. This numerical observation is justified by Proposition 1 .

Proposition 1. Assume that $\left(\lambda_{1 i}, \lambda_{2 i}, \tau_{1}, \tau_{2}, P, t\right)$ with $i \in \mathbb{K}$ is a solution to the optimization problem of Relation (48) corresponding to $\sum_{i \in \mathbb{K}} \lambda_{1 i}=1, \sum_{i \in \mathbb{K}} \lambda_{2 i}=$ 1. Then $\left(\lambda_{1 i}^{\prime}, \lambda_{2 i}^{\prime}, \tau_{1}^{\prime}, \tau_{2}^{\prime}, P, t\right)$ is the solution of the same optimization problem corresponding to $\sum_{i \in \mathbb{K}} \lambda_{1 i}^{\prime}=$ $h_{1}, h_{1}>0, \sum_{i \in \mathbb{K}} \lambda_{2 i}^{\prime}=h_{2}, h_{2}>0$ where $\tau_{1}^{\prime}=h_{1} \tau_{1}$, $\lambda_{1 i}^{\prime}=h_{1} \lambda_{1 i} \tau_{2}^{\prime}=h_{2} \tau_{2}$ and $\lambda_{2 i}^{\prime}=h_{1} \lambda_{2 i}$.

Proof. In the original optimization problem (48) when $h_{1}=h_{2}=1$, we have Relations (49)-(53) as shown in Box I.

The proof follows from the multiplication of Relations (49) and (51) by $h_{1}$, and Relations (50) and (52) by $h_{2}$. Next, by substituting $\tau_{1}^{\prime}=h_{1} \tau_{1}, \lambda_{1 i}^{\prime}=$ $h_{1} \lambda_{1 i} \tau_{2}^{\prime}=h_{2} \tau_{2}$ and $\lambda_{2 i}^{\prime}=h_{1} \lambda_{2 i}$ the set of solutions $\left(\lambda_{1 i}^{\prime}, \lambda_{2 i}^{\prime}, \tau_{1}^{\prime}, \tau_{2}^{\prime}, P, t\right)$ is obtained for the same optimization problem, but for the shifted equality constraints, $\sum_{i \in \mathbb{K}} \lambda_{1 i}^{\prime}=h_{1}, h_{1}>0, \sum_{i \in \mathbb{K}} \lambda_{2 i}^{\prime}=h_{2}, h_{2}>0$.

\section{Examples}

In this section, various examples are introduced to illustrate the previous results. In all cases, the numerical experiments are compared with the proposed method in [20] in terms of the size and volume of the ultimate invariant set of attraction. In this regard, several important points should be considered.

In contrast to the pure LMI-based optimization problems, it is a difficult task to make a fair comparison of BMI-based optimization problems. The reason is that the former belongs to the category of the convex optimization problems for which efficient numerical algorithms handling the practical large-scale problems have been developed. In the convex programming, starting from any initial point, convergence toward a global optimum is always guaranteed and the sole concern is the computational burden which is generally stated in terms of the number of unknown variables and number of LMI inequalities [49]. On the other hand, BMI-based optimization problems are non-convex and seeking a global optimum becomes an NP-hard problem. In most cases, the convergence of various iterative techniques to find a local optimum is not guaranteed mathematically and the choice of initial value is important for convergence to an acceptable solution, which is considered the main weakness of these methods [38]. In the best case, if there exists an algorithm with 


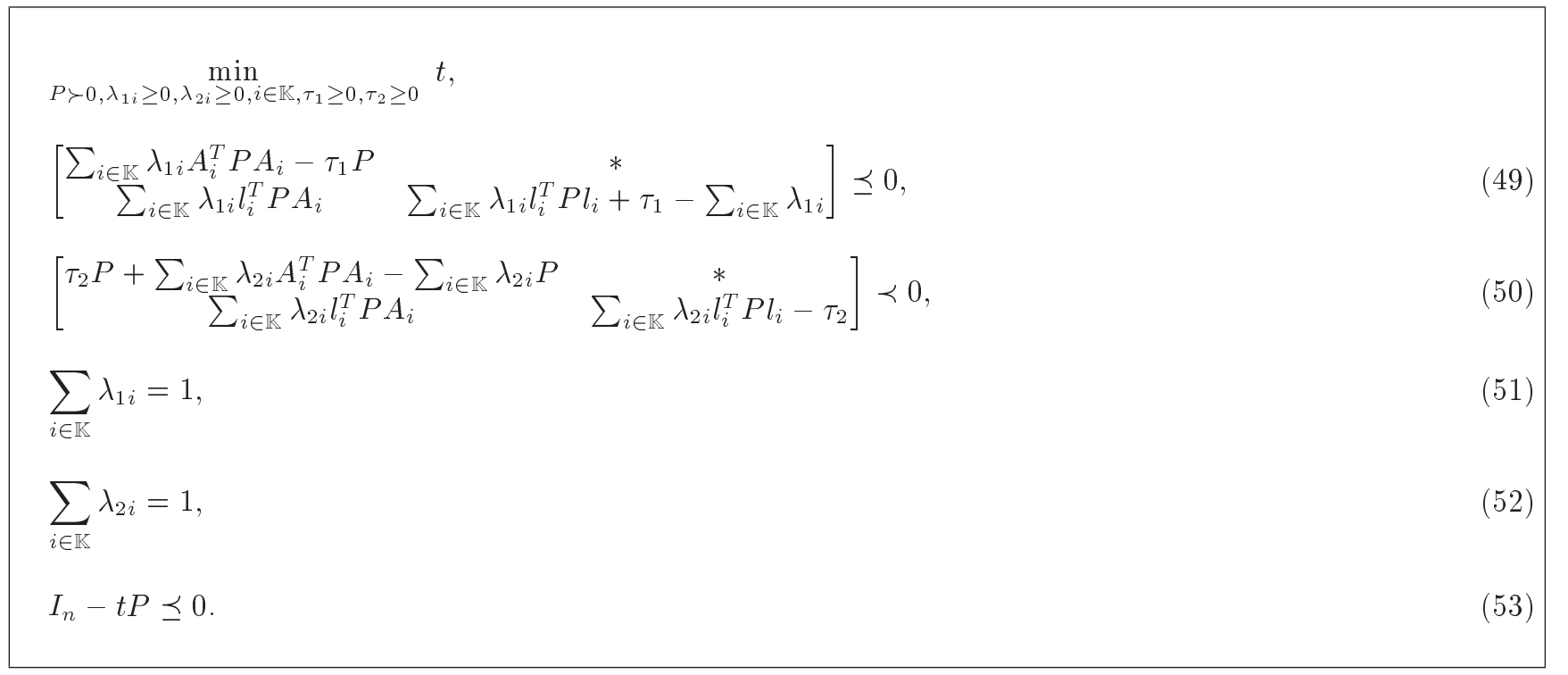

a convergence certificate to a local optimum starting from any initial point, the result will be a local solution merely. In this case, a fundamental question arises as how to compare the local solution of a particular problem formulation with the local solution of another and reach a fair conclusion about the qualities of various problem formulations in terms of the important performance criteria such as conservatism degree.

Nevertheless, our work was compared with Theorem 3 of [20] in terms of the size of the ultimate invariant set of attraction. According to foregoing discussion, it is pointed out that making a rigorous, fair and clear-cut conclusion is a difficult task. In [20], a general shifted quadratic Lyapunov function was used to ensure the global practical stabilization of the discrete-time switched affine systems. While, in our work, a simple centered Lyapunov function was used. The BMI parametrization in [20] used more unknown variables such as vector $h$ and additional matrix variable $W$ that did not exist in our method. In [20], it was assumed that the equilibrium point around which the stabilization was made, belonged to a predetermined set of attainable points. Yet, in our work, such a limitation was relaxed and overlooked. In [20], it was considered necessary that all scalar convex combination variables ( $\lambda$ vector) be fixed and given beforehand, while such a limitation was not an issue in the present work. An attempt was made here to meet the BMI conditions of Theorem 3 in [20] via PENBMI/YALMIP tool without presetting the scalar variables $\lambda_{i}$. However, in all cases PENBMI/YALMIP failed to yield any feasible solution. On the other hand, the proposed conditions in our work were successfully solved and local solutions were extracted in most cases. Intuitively, one reason is that when the parameters $\lambda_{i}$ are not preset on their desirable values, more complex terms including the product of three unknown variables $\lambda_{i} \beta W$ and matrix inverse term $-\sum_{i \in \mathbb{K}} \lambda_{i} l_{i}^{\prime} P A_{i}(I-$ $\left.A_{\lambda}\right)^{-1}$ appear in the conditions of Theorem 3 in [20] which created a large degree of complexity and nonlinearity in the proposed conditions of this theorem. As a result, in all cases, the proposed conditions of Theorem 3 in [20] were solved using the variables $\lambda_{i}$ calculated by the proposed conditions of Theorem 1 in our work. The size of the ultimate invariant set of attraction obtained based on Theorem 3 [20] was statistically, but not in all cases, smaller than that of Theorem 1. This is because, as discussed earlier, the stabilization method in [20] utilizes more unknown variables and therefore, a higher degree of freedom.

Example 1. In the area of power electronics, SingleInductor Multiple-Output (SIMO) converters have attracted much attention due to such advantages as small size and low weight, low cost, great integration, and a wide variety of applications including batteryoperated portable devices, renewable energy systems, and electric vehicles [50]. However, due to the existence of multiple outputs associated with each switching mode, the operation, control, and design of such converters are more challenging than the conventional DC-DC buck and boost converters [51]. In this regard, many modeling and control methods are available in the literature to regulate the SIMO converters of which one can mention model predictive control $[52,53]$, state feedback control [51], multivariable control [54], and digital control [55]. Although a non-averaged model was used to design the controller in [52], the stability of the closed-loop system was not discussed analytically. A common shortcoming of the other works is that 
they have been developed based on the approximate averaged and linearized models by which only local stability of the closed-loop system has been addressed. In this context, for the first time, hybrid modeling and control of power electronics converters were introduced in [56] in which the proposed hybrid control technique was applied to a Single-Inductor Dual-Output (SIDO) converter as well. However, in this work, only the local practical stability of the closed-loop system was addressed by which only the invariant property of a safe ball around a given set point was guaranteed. To the best of our knowledge, the global practical stabilization of the SIMO converters via hybrid control systems theory has not been addressed in the literature.

A DC-DC step-up (boost) SIDO converter is shown in Figure 1. By defining the state vector $x(t)=\left[\begin{array}{lll}i_{L}(t) & v_{A}(t) & v_{B}(t)\end{array}\right]^{T}$, the continuous dynamics associated with each mode are $\dot{x}(t)=A_{c i} x(t)+b_{c i}$, $i \in\{1,2,3\}$ where:

$$
\begin{aligned}
& A_{c 1}=\left[\begin{array}{ccc}
0 & 0 & 0 \\
0 & -\frac{1}{R_{A} C_{A}} & 0 \\
0 & 0 & -\frac{1}{R_{B} C_{B}}
\end{array}\right], \\
& A_{c 2}=\left[\begin{array}{ccc}
0 & -\frac{1}{L} & 0 \\
\frac{1}{C_{A}} & -\frac{1}{R_{A} C_{A}} & 0 \\
0 & 0 & -\frac{1}{R_{B} C_{B}}
\end{array}\right] \text {, } \\
& A_{c 3}=\left[\begin{array}{ccc}
0 & 0 & -\frac{1}{L} \\
0 & \frac{1}{R_{A} C_{A}} & 0 \\
\frac{1}{C_{B}} & 0 & -\frac{1}{R_{B} C_{B}}
\end{array}\right] \text {, } \\
& b_{c 1}=b_{c 2}=b_{c 3}=\left[\begin{array}{lll}
\frac{v_{\text {in }}}{L} & 0 & 0
\end{array}\right]^{T} \text {. }
\end{aligned}
$$

The circuit parameters are $L=500 \mu H, R_{A}=6.25 \Omega$, $R_{B}=34.1 \Omega, C_{A}=800 \mu F, C_{B}=146.6 \mu F$, and $v_{i n}=$ $1.5 \mathrm{~V}$. The sampling time is set to $T_{s}=10 \mu \mathrm{s}$ and as a result the maximum value of switching frequency is limited to $\frac{1}{2 T_{s}}=50 \mathrm{kHz}$. The state-space matrices of the corresponding discrete-time system can be obtained as follows:

$$
A_{i}=e^{A_{c i} T_{s}}, b_{i}=\int_{0}^{T_{s}} e^{A_{c i} t} d t b_{c i},
$$

where $i \in\{1,2,3\}$. The desired output voltages are set to $v_{A d}=1.875 \mathrm{~V}$ and $v_{B d}=3.75 \mathrm{~V}$. The steady-state

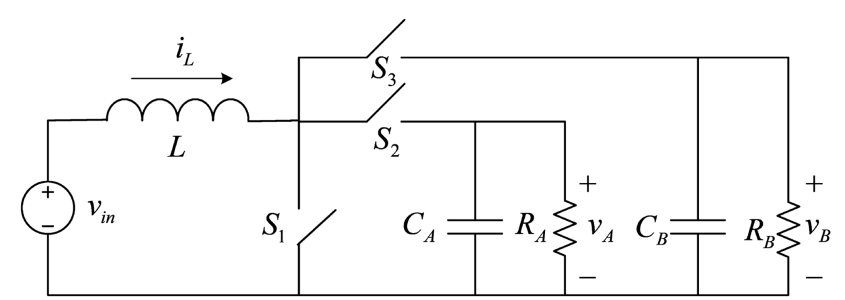

Figure 1. Single-inductor dual-output DC-DC boost converter. current is calculated via the energy balance equation $i_{L d}=\frac{v_{A d}^{2}}{v_{i n} R_{A}}+\frac{v_{B d}^{2}}{v_{i n} R_{B}}$ as $i_{L d}=0.65 A$. The solution of the optimization problem in Relation (48) with $h_{1}=$ $h_{2}=1$ yields $\lambda_{11}=\lambda_{21}=0.3549, \lambda_{12}=\lambda_{22}=0.4867$, $\lambda_{13}=\lambda_{23}=0.1585, \tau_{1}=0.0017, \tau_{2}=0.9983, t_{\min }=$ 1.4128 , with the matrix $P$ as:

$$
P=\left[\begin{array}{ccc}
1.6886 & -0.0822 & -0.1146 \\
-0.0822 & 3.3647 & -0.4949 \\
-0.1146 & -0.4949 & 0.8172
\end{array}\right]
$$

The invariant set of attraction as well as the state trajectories $x(k)$ corresponding to various initial conditions are shown in Figure 2. Figure 3 illustrates the

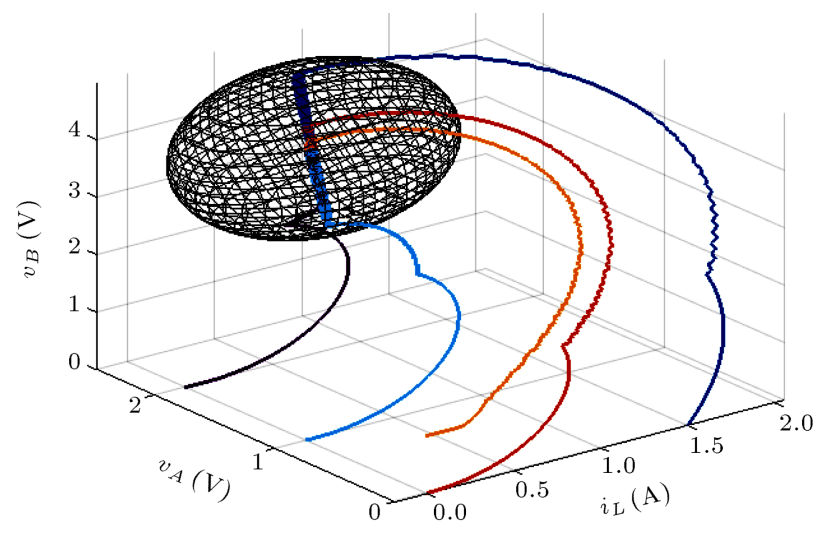

Figure 2. State trajectories and invariant set of attraction for the Single Inductor Dual-Output (SIDO) DC-DC boost converter.

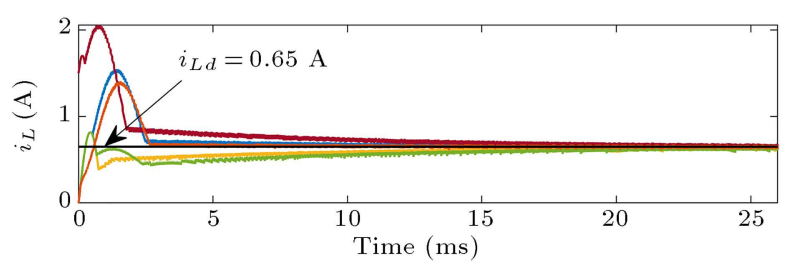

(a)

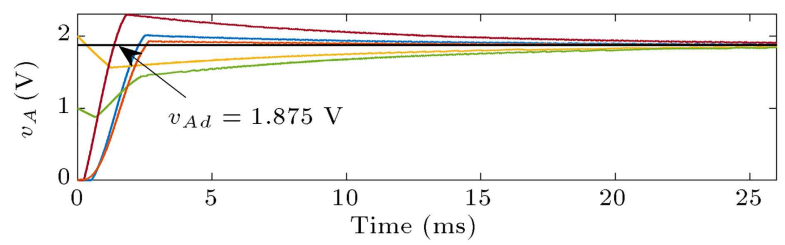

(b)

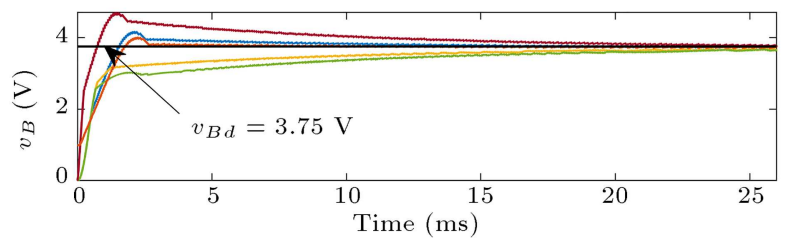

(c)

Figure 3. Time profiles of the Single Inductor Dual-Output (SIDO) DC-DC boost converter states: (a) Inductor current, (b) output voltage $v_{A}$, and (c) output voltage $v_{B}$. 
Table 1. Ellipsoid size comparisons for the DC-DC Single Inductor Dual-Output (SIDO) boost converter.

\begin{tabular}{|c|c|c|c|c|c|}
\hline $\begin{array}{c}\text { Comparison } \\
\text { quantities }\end{array}$ & $\begin{array}{l}\text { Theorem } 1 \\
\text { ellipsoid size }\end{array}$ & $\begin{array}{c}\text { Theorem } 3 \text { of }[20] \\
\lambda_{1}=\frac{\lambda_{21}}{\sum_{i \in \mathbb{K}} \lambda_{2 i}} \\
\lambda_{2}=\frac{\lambda_{22}}{\sum_{i \in \mathbb{K}} \lambda_{2 i}} \\
\lambda_{3}=\frac{\lambda_{23}}{\sum_{i \in \mathbb{K}} \lambda_{2 i}} \\
\text { ellipsoid size }\end{array}$ & $\begin{array}{c}\text { Theorem } 1 \\
\sum_{i \in \mathbb{K}} \lambda_{1 i}=1 \\
\sum_{i \in \mathbb{K}} \lambda_{2 i}=1\end{array}$ & $\begin{array}{c}\text { Theorem } 1 \\
\sum_{i \in \mathbb{K}} \lambda_{1 i}>0 \\
\sum_{i \in \mathbb{K}} \lambda_{2 i}=1\end{array}$ & $\begin{array}{c}\text { Theorem } 1 \\
\sum_{i \in \mathbb{K}} \lambda_{1 i}=1 \\
\sum_{i \in \mathbb{K}} \lambda_{2 i}>0\end{array}$ \\
\hline$t$ & 1.4128 & 1.4323 & $\lambda_{11}=0.3549$ & $\lambda_{11}=6.9319 \cdot 10^{-6}$ & $\lambda_{11}=0.3549$ \\
\hline \multirow{5}{*}{$\frac{1}{\sqrt{\operatorname{det}(P)}}$} & 0.4711 & 0.4897 & $\lambda_{12}=0.4867$ & $\lambda_{12}=9.5271 \cdot 10^{-6}$ & $\lambda_{12}=0.4867$ \\
\hline & & & $\lambda_{13}=0.1585$ & $\lambda_{13}=3.0909 \cdot 10^{-6}$ & $\lambda_{13}=0.1585$ \\
\hline & & & $\lambda_{21}=0.3549$ & $\lambda_{21}=0.3549$ & $\lambda_{21}=0.0278$ \\
\hline & & & $\lambda_{22}=0.4867$ & $\lambda_{22}=0.4867$ & $\lambda_{22}=0.0381$ \\
\hline & & & $\lambda_{23}=0.1585$ & $\lambda_{23}=0.1585$ & $\lambda_{23}=0.0124$ \\
\hline
\end{tabular}
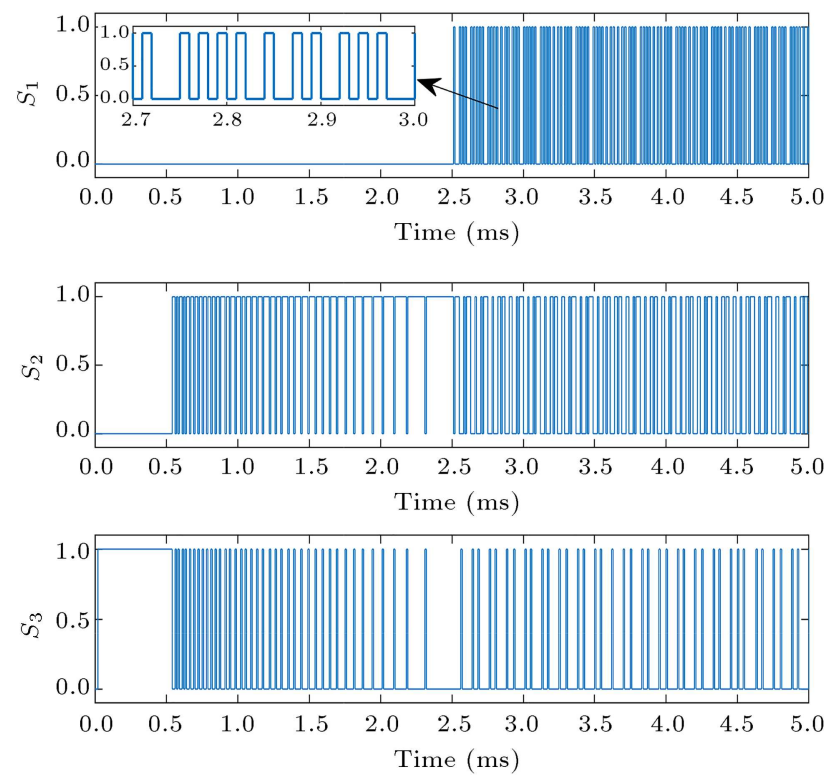

Figure 4. Switching signals of the Single Inductor Dual-Output (SIDO) DC-DC boost converter starting from an initial condition at the origin.

corresponding time simulations and Figure 4 shows the switching signals corresponding to the initial condition in the origin.

For the specified operating point, the results of the comparison between the conditions of Theorem 1 and Theorem 3 in [20] are given in Table 1 . As discussed earlier, the BMI conditions of Theorem 3 in [20] are solved via values of $\lambda_{2 i}$ obtained by solving the conditions of Theorem 1. It should be noted that through this selection, matrix $A_{\lambda}$ is Schur stable and these preset values satisfy the BMI conditions of Theorem 3 in [20]. This result is confirmed by Corollary 1. The parameter $\frac{1}{\sqrt{\operatorname{det}(P)}}$, in Table 1 is proportional to the volume of the respective ellipsoid as the ultimate invariant set of attraction and the parameter $t$ is an upper bound to the square of the maximal semiaxis of the same ellipsoid.
As discussed in Section 4, to achieve suitable and acceptable results of the PENBMI software and to obtain less conservative solutions, different versions of Constraints (8) and (9) of Theorem 1 were employed. However, according to Table 1, in all cases, the obtained optimal solutions were identical and only the scalar variables $\lambda_{1 i}$ and $\lambda_{2 i}$ varied. This behavior was detected in other examples, as well. Moreover, in this particular example and at an operating point, the optimal solutions of Theorem 1 outperformed those of Theorem 3 in [20].

Example 2. The second example is an academic one borrowed from [20]. To obtain acceptable solutions from PENBMI software, here, we needed to choose a shorter discretization time than $T_{s}=1$ in [20]. In this comparative study, $T_{s}=0.05$ was chosen to obtain acceptable results in a wide range of operating points. Obviously, at some operating points, we could choose larger values of $T_{s}$ however, to unify the numerical results, we selected a common discretization time as $T_{s}=0.05$. The discrete-time switched affine system consisting of 2 unstable systems defined by the following state space matrices is considered 2 as follows:

$$
\begin{aligned}
& A_{c 1}=\left[\begin{array}{ccc}
0 & 1 & 0 \\
0 & 0 & 1 \\
-1 & -1 & -1
\end{array}\right], \\
& A_{c 2}=\left[\begin{array}{ccc}
0 & 1 & 0 \\
0 & 0 & 1 \\
0 & -1 & -1
\end{array}\right], \\
& b_{c 1}=\left[\begin{array}{lll}
1 & 0 & 1
\end{array}\right]^{T}, \quad b_{c 2}=\left[\begin{array}{lll}
0 & 1 & 0
\end{array}\right]^{T} .
\end{aligned}
$$

The discrete-time system matrices $A_{i}$ and $b_{i}, i \in\{1,2\}$ are calculated through Eq. (55). Table 2 gives the parameters related to the size of ellipsoids obtained by solving the conditions of Theorem 1 and Theorem 3 in [20]. In this table, the symbol "_" denotes that no convergence is achieved in the respective case. 
Table 2. Ellipsoid size comparisons for numerical Example 2.

\begin{tabular}{|c|c|c|c|c|c|c|}
\hline $\begin{array}{l}\text { Operating } \\
\text { point }\end{array}$ & $\begin{array}{c}\text { Comparison } \\
\text { quantities }\end{array}$ & $\begin{array}{l}\text { Theorem } 1 \\
\text { ellipsoid size }\end{array}$ & $\begin{array}{c}\text { Theorem } 3 \text { of }[20] \\
\lambda_{1}=\frac{\lambda_{21}}{\sum_{i \in \mathbb{K}} \lambda_{2 i}} \\
\lambda_{2}=\frac{\lambda_{22}}{\sum_{i \in \mathbb{K}} \lambda_{2 i}} \\
\lambda_{3}=\frac{\lambda_{23}}{\sum_{i \in \mathbb{K}} \lambda_{2 i}} \\
\text { ellipsoid size }\end{array}$ & $\begin{array}{c}\text { Theorem } 1 \\
\sum_{i \in \mathbb{K}} \lambda_{1 i}=1 \\
\sum_{i \in \mathbb{K}} \lambda_{2 i}=1\end{array}$ & $\begin{array}{c}\text { Theorem } 1 \\
\sum_{i \in \mathbb{K}} \lambda_{1 i}=1 \\
\sum_{i \in \mathbb{K}} \lambda_{2 i}>0\end{array}$ & $\begin{array}{c}\text { Theorem } 1 \\
\sum_{i \in \mathbb{K}} \lambda_{1 i}>0 \\
\sum_{i \in \mathbb{K}} \lambda_{2 i}=1\end{array}$ \\
\hline \multirow[t]{2}{*}{$p=0.1$} & $t$ & - & - & \multirow[t]{2}{*}{-} & \multirow[t]{2}{*}{-} & \multirow[t]{2}{*}{-} \\
\hline & $\frac{1}{\sqrt{\operatorname{det}(P)}}$ & - & - & & & \\
\hline \multirow{4}{*}{$p=0.2$} & $t$ & 3.9544 & 4.7667 & \multirow{4}{*}{$\begin{array}{l}\lambda_{11}=0.2085 \\
\lambda_{12}=0.7915 \\
\lambda_{21}=0.2085 \\
\lambda_{22}=0.7915\end{array}$} & $\lambda_{11}=0.2085$ & $\lambda_{11}=0.0410$ \\
\hline & $\frac{1}{\sqrt{\operatorname{det}(P)}}$ & 2.5631 & 3.5505 & & $\lambda_{12}=0.7915$ & $\lambda_{12}=0.1556$ \\
\hline & & & & & $\lambda_{21}=0.0325$ & $\lambda_{21}=0.2085$ \\
\hline & & & & & $\lambda_{22}=0.1234$ & $\lambda_{22}=0.7915$ \\
\hline \multirow{4}{*}{$p=0.3$} & $t$ & 2.0630 & 2.1559 & \multirow{4}{*}{$\begin{array}{l}\lambda_{11}=0.3035 \\
\lambda_{12}=0.6965 \\
\lambda_{21}=0.3035 \\
\lambda_{22}=0.6965\end{array}$} & $\lambda_{11}=0.3035$ & \multirow[t]{4}{*}{ - } \\
\hline & $\frac{1}{\sqrt{\operatorname{det}(P)}}$ & 1.0428 & 1.1754 & & $\lambda_{12}=0.6965$ & \\
\hline & & & & & $\lambda_{21}=0.0134$ & \\
\hline & & & & & $\lambda_{22}=0.0309$ & \\
\hline \multirow[t]{2}{*}{$p=0.4$} & $t$ & 1.3561 & 1.2391 & \multirow{2}{*}{$\begin{array}{l}\lambda_{11}=0.3984 \\
\lambda_{12}=0.6965 \\
\lambda_{21}=0.3984 \\
\lambda_{22}=0.6016\end{array}$} & \multirow[t]{2}{*}{-} & \multirow[t]{2}{*}{-} \\
\hline & $\frac{1}{\sqrt{\operatorname{det}(P)}}$ & 0.5948 & 0.5484 & & & \\
\hline \multirow[t]{2}{*}{$p=0.5$} & $t$ & 1.0054 & 0.8429 & \multirow{2}{*}{$\begin{array}{l}\lambda_{11}=0.4950 \\
\lambda_{12}=0.5050 \\
\lambda_{21}=0.4950 \\
\lambda_{22}=0.5050\end{array}$} & \multirow[t]{2}{*}{-} & \multirow[t]{2}{*}{-} \\
\hline & $\frac{1}{\sqrt{\operatorname{det}(P)}}$ & 0.4008 & 0.3182 & & & \\
\hline \multirow[t]{4}{*}{$p=0.6$} & $t$ & 0.8038 & 0.6440 & \multirow{2}{*}{$\begin{array}{l}\lambda_{11}=0.5936 \\
\lambda_{12}=0.4064\end{array}$} & \multirow[t]{4}{*}{-} & $\lambda_{11}=0.1030$ \\
\hline & $\frac{1}{\sqrt{\operatorname{det}(P)}}$ & 0.2985 & 0.2171 & & & $\lambda_{12}=0.0706$ \\
\hline & & & & $\lambda_{21}=0.5936$ & & $\lambda_{21}=0.5936$ \\
\hline & & & & $\lambda_{22}=0.4064$ & & $\lambda_{22}=0.4064$ \\
\hline \multirow[t]{4}{*}{$p=0.7$} & $t$ & 0.6776 & 0.5448 & \multirow{4}{*}{$\begin{array}{l}\lambda_{11}=0.6937 \\
\lambda_{12}=0.3063 \\
\lambda_{21}=0.6937 \\
\lambda_{22}=0.3063\end{array}$} & $\lambda_{11}=0.6937$ & $\lambda_{11}=0.0651$ \\
\hline & $\frac{1}{\sqrt{\operatorname{det}(P)}}$ & 0.2384 & 0.1721 & & $\lambda_{12}=0.3063$ & $\lambda_{12}=0.0288$ \\
\hline & & & & & $\lambda_{21}=0.1076$ & $\lambda_{21}=0.6937$ \\
\hline & & & & & $\lambda_{22}=0.0475$ & $\lambda_{22}=0.3063$ \\
\hline \multirow[t]{2}{*}{$p=0.8$} & $t$ & 0.5955 & 0.4665 & \multirow{2}{*}{$\begin{array}{l}\lambda_{11}=0.7952 \\
\lambda_{12}=0.2048 \\
\lambda_{21}=0.7952 \\
\lambda_{22}=0.2048\end{array}$} & \multirow[t]{2}{*}{-} & \multirow[t]{2}{*}{-} \\
\hline & $\frac{1}{\sqrt{\operatorname{det}(P)}}$ & 0.2020 & 0.1369 & & & \\
\hline \multirow[t]{2}{*}{$p=0.9$} & $t$ & 0.5431 & 0.4280 & \multirow{2}{*}{$\begin{array}{l}\lambda_{11}=0.8974 \\
\lambda_{12}=0.1026 \\
\lambda_{21}=0.8974 \\
\lambda_{22}=0.1026\end{array}$} & \multirow[t]{2}{*}{-} & \multirow[t]{2}{*}{-} \\
\hline & $\frac{1}{\sqrt{\operatorname{det}(P)}}$ & 0.1831 & 0.1247 & & & \\
\hline
\end{tabular}




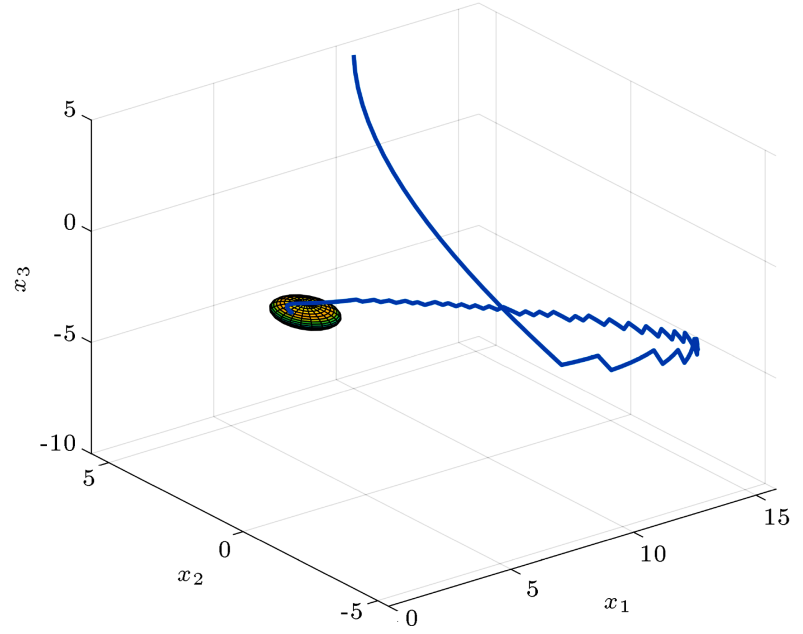

Figure 5. State $x$ trajectory and invariant set of attraction.

Again, different versions of Constraints (8) and (9) of Theorem 1 are employed. Nevertheless, according to Table 2, in all cases where convergence is achieved, the obtained optimal solutions are identical and only the scalar variables $\lambda_{1 i}$ and $\lambda_{2 i}$ vary. This behavior is observed in the following examples, as well. Therefore, in the next examples, we only consider the numerically well-behaved versions of original Constraints (8) and (9) in Theorem 1 as $\sum_{i \in \mathbb{K}} \lambda_{2 i}=1$ and $\sum_{i \in \mathbb{K}} \lambda_{1 i}=$ 1. According to Table 2, at the operating points corresponding to $\lambda=0.2$ and $\lambda=0.3$, Theorem 1 yields less conservative results, while in other cases, Theorem 3 of [20] yields better responses.

By starting from $x_{0}=\left[\begin{array}{lll}10 & 5 & 5\end{array}\right]^{T}$ and setting $p=0.5$, Figure 5 shows the state trajectory $x(k)$ and invariant set of attraction. The time evolution of the trajectories $x(k)$ and switching function are shown in Figure 6.

In the sequel, we compare our methodology with Theorem 3 in [20] in the case of the basic and classical DC-DC boost, buck and buck-boost converters. The parameters used for the numerical experiments are given in Table 3 [57]. To avoid ill-conditioned matrix inequalities and make the problem more amenable for numerical purposes, per unit parameters are used in the numerical experiments. The base parameters are selected as $v_{\text {base }}=50 \mathrm{~V}, i_{\text {base }}=2.5 \mathrm{~A}$, and $T_{\text {base }}=10 \mu \mathrm{s}$.

Table 3. The specifications of the DC-DC boost, buck and buck-boost converters [57].

\begin{tabular}{ccc}
\hline Input voltage, $v_{s}$ & $50 \mathrm{~V}$ & $1 \mathrm{p} . u$. \\
Converter inductor, $L$ & $2 \mathrm{mH}$ & $10 \mathrm{p} . u$. \\
Output capacitor, $C$ & $100 \mu \mathrm{F}$ & 200 p.u. \\
Load resistance, $R$ & $50 \Omega$ & 2.5 p.u. \\
Sampling period, $T_{s}$ & $10 \mu \mathrm{s}$ & 1 p.u. \\
Inductor series resistance, $r_{L}$ & $0.5 \Omega$ & 0.025 p.u. \\
Capacitor series resistance, $r_{C}$ & $0.1 \Omega$ & 0.005 p.u. \\
\hline
\end{tabular}
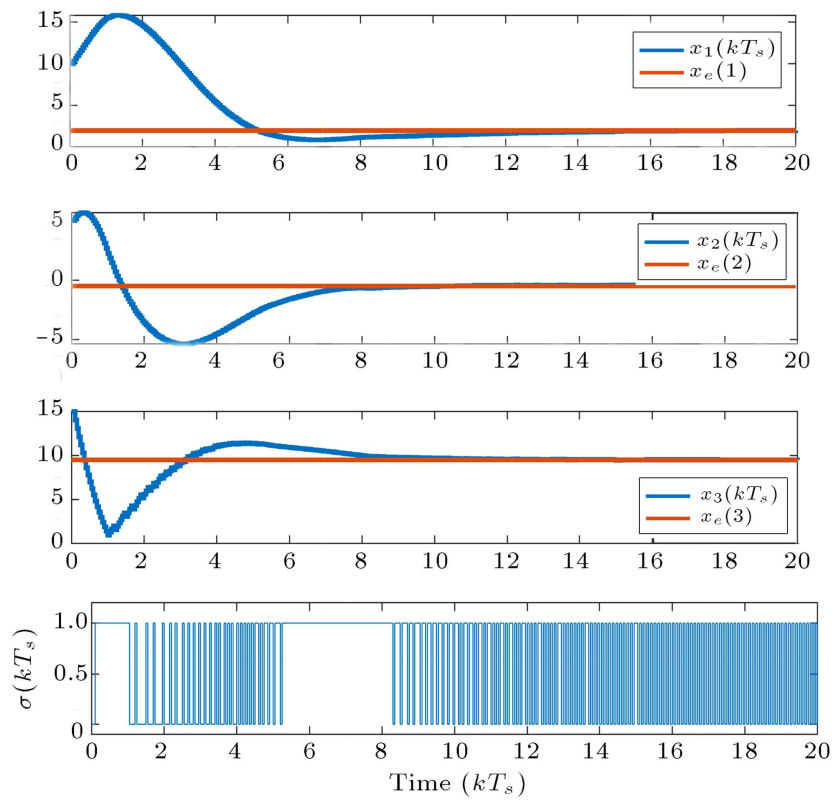

Figure 6. Time profiles of the trajectories $x$ and switching function $\sigma$.

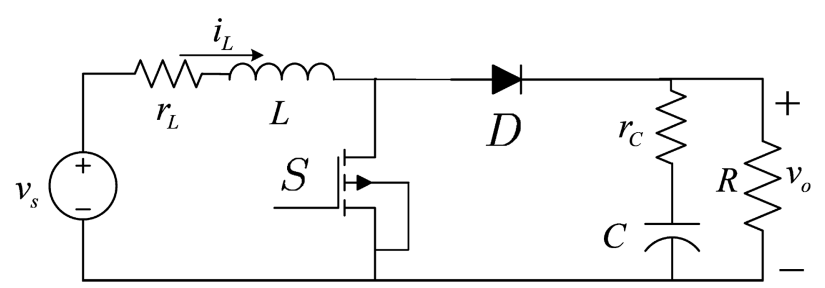

Figure 7. DC-DC boost converter.

Example 3. Figure 7 shows the schematic diagram of a DC-DC boost converter. The continuous states of the system are defined as $x(t)=\left[i_{L}(t), v_{o}(t)\right]^{T}$ which are the inductor current and converter output voltage, respectively. The state-space equations of this converter are as follows $[58,59]$ :

$$
\begin{aligned}
& \dot{x}(t)=A_{c 1} x(t)+b_{c 1}, \\
& \dot{x}(t)=A_{c 2} x(t)+b_{c 2},
\end{aligned}
$$

where:

$$
\begin{aligned}
& A_{c 1}=\left[\begin{array}{cc}
-\frac{r_{L}}{L} & 0 \\
0 & -\frac{1}{C} \frac{1}{\left(R+r_{C}\right) C}
\end{array}\right], \quad b_{c 1}=\left[\begin{array}{c}
\frac{v_{s}}{L} \\
0
\end{array}\right], \\
& A_{c 2}=\left[\begin{array}{cc}
\frac{R}{R+r_{C}}\left(\frac{1}{C}-\frac{r_{L}}{L}-\frac{r_{C} r_{L}}{L}\right) & -\frac{R}{R+r_{C}}\left(\frac{1}{L}+\frac{1}{L}\right)
\end{array}\right], \\
& b_{c 2}=\left[\begin{array}{c}
\frac{v_{s}}{L} \\
\frac{v_{s}}{R+r_{C}} \frac{r_{C}}{L} v_{s}
\end{array}\right] .
\end{aligned}
$$

Discrete-time state equations can be obtained via Eq. (55). Table 4 gives the size of the invariant 
Table 4. Ellipsoid size comparisons for the DC-DC boost converter.

\begin{tabular}{|c|c|c|c|c|}
\hline $\begin{array}{l}\text { Operating } \\
\text { point }\end{array}$ & $\begin{array}{c}\text { Comparison } \\
\text { quantities }\end{array}$ & $\begin{array}{l}\text { Theorem } 1 \\
\text { ellipsoid size }\end{array}$ & $\begin{array}{c}\text { Theorem } 3 \text { of }[20] \\
\lambda_{1}=\frac{\lambda_{21}}{\sum_{i \in \mathbb{K}} \lambda_{2 i}} \\
\lambda_{2}=\frac{\lambda_{22}}{\sum_{i \in \mathbb{K}} \lambda_{2 i}} \\
\text { ellipsoid size }\end{array}$ & $\begin{array}{c}\text { Theorem } 1 \\
\sum_{i \in \mathbb{K}} \lambda_{1 i}=1 \\
\sum_{i \in \mathbb{K}} \lambda_{2 i}=1\end{array}$ \\
\hline \multirow[t]{2}{*}{$p=0.1$} & $t$ & 0.2522 & 0.2281 & \multirow{2}{*}{$\begin{array}{l}\lambda_{11}=0.0997 \\
\lambda_{12}=0.9003 \\
\lambda_{21}=0.0997 \\
\lambda_{22}=0.9003\end{array}$} \\
\hline & $\frac{1}{\sqrt{\operatorname{det}(P)}}$ & 0.0562 & 0.0509 & \\
\hline \multirow[t]{2}{*}{$p=0.2$} & $t$ & 0.5965 & 0.5249 & \multirow{2}{*}{$\begin{array}{l}\lambda_{11}=0.1997 \\
\lambda_{12}=0.8003 \\
\lambda_{21}=0.1997 \\
\lambda_{22}=0.8003\end{array}$} \\
\hline & $\frac{1}{\sqrt{\operatorname{det}(P)}}$ & 0.1331 & 0.1172 & \\
\hline \multirow[t]{2}{*}{$p=0.3$} & $t$ & 1.0526 & 0.9163 & \multirow{2}{*}{$\begin{array}{l}\lambda_{11}=0.2997 \\
\lambda_{12}=0.7003 \\
\lambda_{21}=0.2997 \\
\lambda_{22}=0.7003\end{array}$} \\
\hline & $\frac{1}{\sqrt{\operatorname{det}(P)}}$ & 1.0428 & 0.2351 & \\
\hline \multirow[t]{2}{*}{$p=0.4$} & $t$ & 1.6588 & 1.4468 & \multirow{2}{*}{$\begin{array}{l}\lambda_{11}=0.3998 \\
\lambda_{12}=0.6002 \\
\lambda_{21}=0.3998 \\
\lambda_{22}=0.6002\end{array}$} \\
\hline & $\frac{1}{\sqrt{\operatorname{det}(P)}}$ & 0.3709 & 0.3231 & \\
\hline \multirow[t]{2}{*}{$p=0.5$} & $t$ & 2.4775 & 2.1918 & \multirow{2}{*}{$\begin{array}{l}\lambda_{11}=0.4999 \\
\lambda_{12}=0.5001 \\
\lambda_{21}=0.4999 \\
\lambda_{22}=0.5001\end{array}$} \\
\hline & $\frac{1}{\sqrt{\operatorname{det}(P)}}$ & 0.5550 & 0.4895 & \\
\hline \multirow[t]{2}{*}{$p=0.6$} & $t$ & 3.6101 & 3.2869 & \multirow{2}{*}{$\begin{array}{l}\lambda_{11}=0.6000 \\
\lambda_{12}=0.4000 \\
\lambda_{21}=0.6000 \\
\lambda_{22}=0.4000\end{array}$} \\
\hline & $\frac{1}{\sqrt{\operatorname{det}(P)}}$ & 0.8110 & 0.7343 & \\
\hline \multirow[t]{2}{*}{$p=0.7$} & $t$ & 5.2074 & 4.9806 & \multirow{2}{*}{$\begin{array}{l}\lambda_{11}=0.7000 \\
\lambda_{12}=0.3000 \\
\lambda_{21}=0.7000 \\
\lambda_{22}=0.3000\end{array}$} \\
\hline & $\frac{1}{\sqrt{\operatorname{det}(P)}}$ & 1.1759 & 1.1142 & \\
\hline \multirow[t]{2}{*}{$p=0.8$} & $t$ & 7.3251 & 7.6248 & \multirow{2}{*}{$\begin{array}{l}\lambda_{11}=0.8000 \\
\lambda_{12}=0.2000 \\
\lambda_{21}=0.8000 \\
\lambda_{22}=0.2000\end{array}$} \\
\hline & $\frac{1}{\sqrt{\operatorname{det}(P)}}$ & 1.6724 & 1.7116 & \\
\hline \multirow[t]{2}{*}{$p=0.9$} & $t$ & 7.9900 & 9.1003 & \multirow{2}{*}{$\begin{array}{l}\lambda_{11}=0.8999 \\
\lambda_{12}=0.1001 \\
\lambda_{21}=0.8999 \\
\lambda_{22}=0.1001\end{array}$} \\
\hline & $\frac{1}{\sqrt{\operatorname{det}(P)}}$ & 1.9520 & 2.3733 & \\
\hline
\end{tabular}




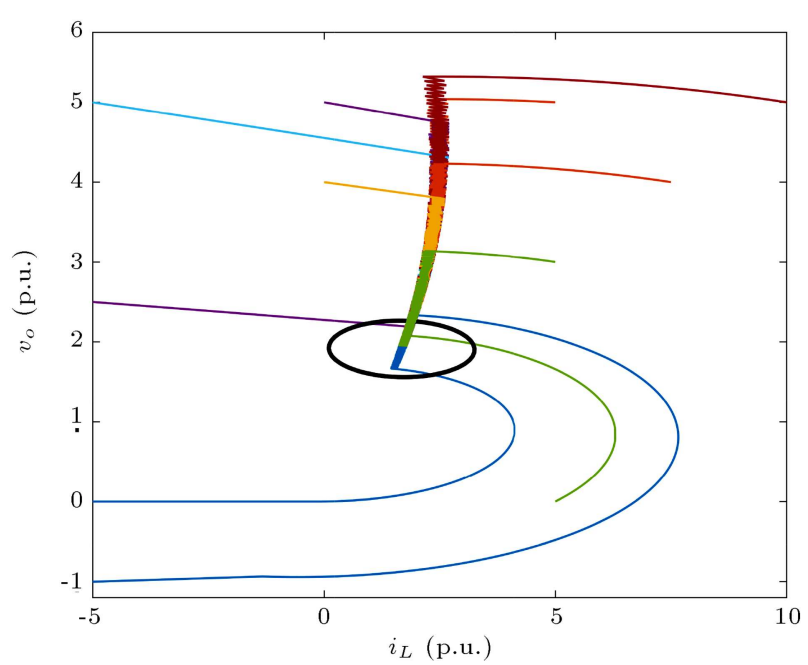

Figure 8. Invariant set of attraction and state trajectories of the DC-DC boost converter corresponding to the operating point $p=0.5$.
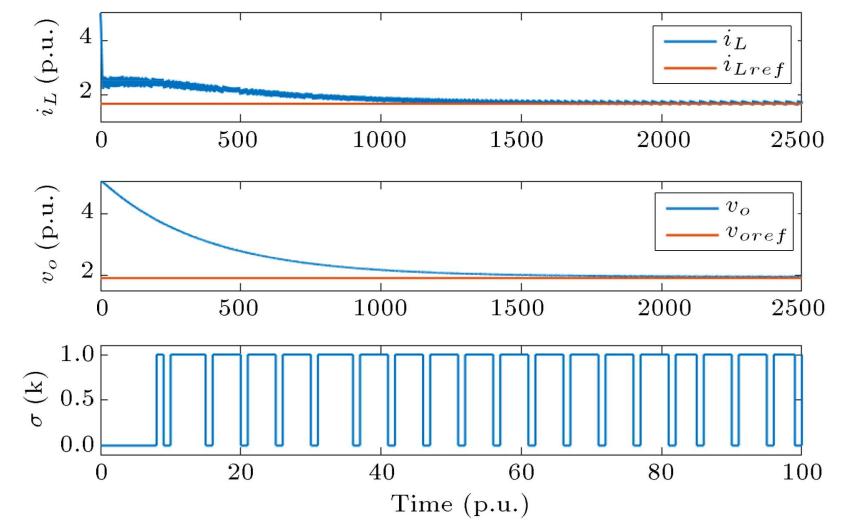

Figure 9. Time evolution of the state variables and switching function for the DC-DC boost converter.

set of attraction by solving conditions of Theorem 1 and Theorem 3 in [20]. According to this table, at the operating points corresponding to $p=0.1-0.7$, Theorem 3 of [20] results in less conservative results, while at the operating points corresponding to $p=$ $0.8-0.9$, Theorem 1 gives smaller sizes.

The state trajectories $x(k)=\left[i_{L}(k), v_{o}(k)\right]^{T}$ starting from various initial points and invariant set of attraction corresponding to the operating point $p=0.5$ are shown in Figure 8. Figure 9 illustrates the time evolutions of the state variables and switching sequence corresponding to the initial condition $i_{L}(0)=v_{o}(0)=$ 5 p.u.

Example 4. Figure 10 shows the circuit diagram of a DC-DC buck converter. Let the continuous states of the system be defined as $x(t)=\left[i_{L}(t), v_{o}(t)\right]^{T}$ which are the inductor current and converter output voltage respectively. The state-space equations of the converter are given as Eq. (58) where [60,61]:

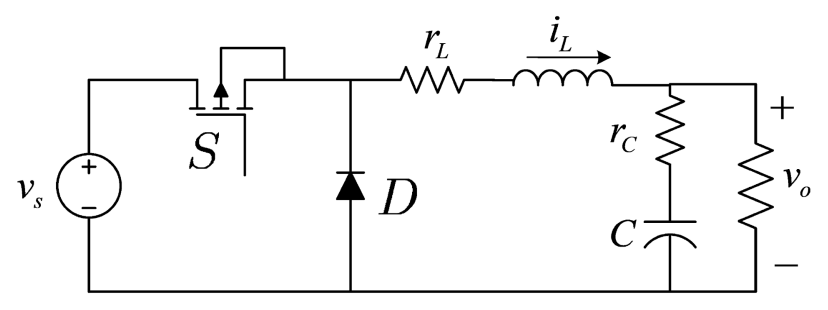

Figure 10. DC-DC buck converter.

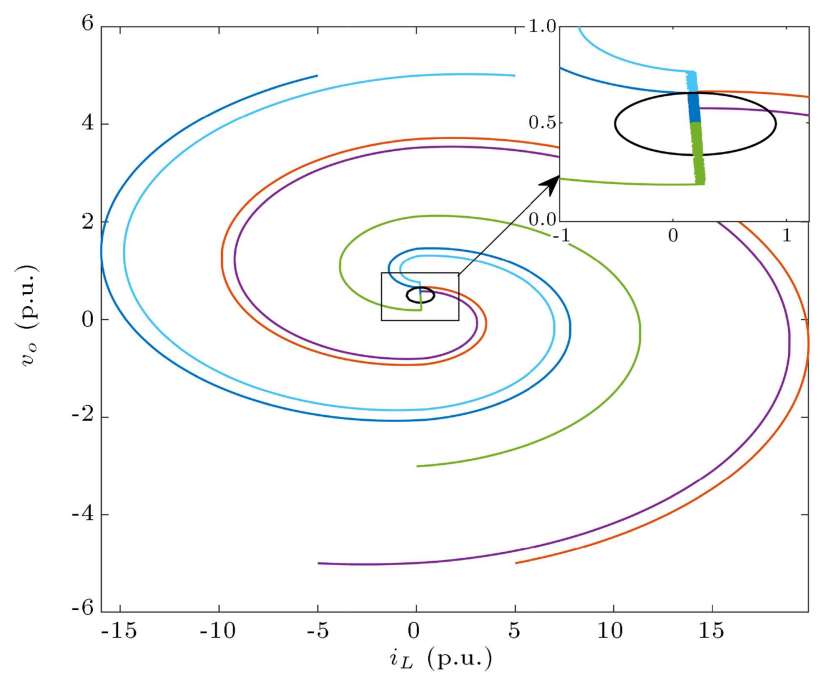

Figure 11. Invariant set of attraction and state trajectories of the DC-DC buck converter corresponding to the operating point $p=0.5$.

$$
\begin{aligned}
A_{c 1} & =A_{c 2} \\
& =\left[\begin{array}{ll}
\frac{1}{C} \frac{R}{R+r_{C}}\left(1-\frac{r_{L}}{L}\right. \\
\left.C r_{C} \frac{r_{L}}{L}\right) & -\frac{1}{C} \frac{1}{R+r_{C}}\left(1+C r_{C} \frac{R}{L}\right)
\end{array}\right], \\
b_{c 1} & =\left[\begin{array}{ll}
\frac{v_{s}}{L} \\
v_{s} \frac{R}{R+r_{C}} \frac{r_{C}}{L}
\end{array}\right], \\
b_{c 2} & =\left[\begin{array}{l}
0 \\
0
\end{array}\right] .
\end{aligned}
$$

Table 5 gives the size of the ellipsoids by solving the BMI conditions of Theorem 1 and Theorem 3 in [20]. As it can be seen from this table, in the case of DC-DC buck converter, both theorems yield identical results. The state trajectory $x(k)$ starting from various initial states and the invariant set of attraction corresponding to the operating point $p=0.5$ are shown in Figure 11 . Figure 12, illustrates the time evolutions of the state variables and switching function corresponding to the initial condition $i_{L}(0)=v_{o}(0)=5$ p.u.

Example 5. Figure 13 shows the schematic diagram of a DC-DC buck-boost converter. Considering $x(t)=$ $\left[i_{L}(t), v_{o}(t)\right]^{T}$, the state-space equations are given as 
Table 5. Ellipsoid size comparisons for the DC-DC buck converter.

\begin{tabular}{|c|c|c|c|c|}
\hline $\begin{array}{l}\text { Operating } \\
\text { point }\end{array}$ & $\begin{array}{l}\text { Comparison } \\
\text { quantities }\end{array}$ & $\begin{array}{l}\text { Theorem } 1 \\
\text { ellipsoid size }\end{array}$ & $\begin{array}{c}\text { Theorem } 3 \text { of }[20] \\
\lambda_{1}=\frac{\lambda_{21}}{\sum_{i \in \mathbb{K}} \lambda_{2 i}} \\
\lambda_{2}=\frac{\lambda_{22}}{\sum_{i \in \mathbb{K}} \lambda_{2 i}} \\
\text { ellipsoid size }\end{array}$ & $\begin{array}{c}\text { Theorem } 1 \\
\sum_{i \in \mathbb{K}} \lambda_{1 i}=1 \\
\sum_{i \in \mathbb{K}} \lambda_{2 i}=1\end{array}$ \\
\hline \multirow[t]{2}{*}{$p=0.1$} & $t$ & 0.1803 & 0.1803 & \multirow{2}{*}{$\begin{array}{l}\lambda_{11}=0.1000 \\
\lambda_{12}=0.9000 \\
\lambda_{21}=0.1000 \\
\lambda_{22}=0.9000\end{array}$} \\
\hline & $\frac{1}{\sqrt{\operatorname{det}(P)}}$ & 0.0402 & 0.0402 & \\
\hline \multirow[t]{2}{*}{$p=0.2$} & $t$ & 0.3205 & 0.3205 & \multirow{2}{*}{$\begin{array}{l}\lambda_{11}=0.2000 \\
\lambda_{12}=0.8000 \\
\lambda_{21}=0.2000 \\
\lambda_{22}=0.8000\end{array}$} \\
\hline & $\frac{1}{\sqrt{\operatorname{det}(P)}}$ & 0.0715 & 0.0715 & \\
\hline \multirow{2}{*}{$p=0.3$} & $t$ & 0.4206 & 0.4206 & \multirow{2}{*}{$\begin{array}{l}\lambda_{11}=0.3000 \\
\lambda_{12}=0.7000 \\
\lambda_{21}=0.3000 \\
\lambda_{22}=0.7000\end{array}$} \\
\hline & $\frac{1}{\sqrt{\operatorname{det}(P)}}$ & 0.0938 & 0.0938 & \\
\hline \multirow[t]{2}{*}{$p=0.4$} & $t$ & 0.4807 & 0.4807 & \multirow{2}{*}{$\begin{array}{l}\lambda_{11}=0.4000 \\
\lambda_{12}=0.6000 \\
\lambda_{21}=0.4000 \\
\lambda_{22}=0.6000\end{array}$} \\
\hline & $\frac{1}{\sqrt{\operatorname{det}(P)}}$ & 0.1072 & 0.1072 & \\
\hline \multirow[t]{2}{*}{$p=0.5$} & $t$ & 0.5007 & 0.5007 & \multirow{2}{*}{$\begin{array}{l}\lambda_{11}=0.5000 \\
\lambda_{12}=0.5000 \\
\lambda_{21}=0.5000 \\
\lambda_{22}=0.5000\end{array}$} \\
\hline & $\frac{1}{\sqrt{\operatorname{det}(P)}}$ & 0.1117 & 0.1117 & \\
\hline \multirow[t]{2}{*}{$p=0.6$} & $t$ & 0.4807 & 0.4807 & \multirow{2}{*}{$\begin{array}{l}\lambda_{11}=0.6000 \\
\lambda_{12}=0.4000 \\
\lambda_{21}=0.6000 \\
\lambda_{22}=0.4000\end{array}$} \\
\hline & $\frac{1}{\sqrt{\operatorname{det}(P)}}$ & 0.1072 & 0.1072 & \\
\hline \multirow[t]{2}{*}{$p=0.7$} & $t$ & 0.4206 & 0.4206 & \multirow[b]{2}{*}{$\begin{array}{l}\lambda_{11}=0.7000 \\
\lambda_{12}=0.3000 \\
\lambda_{21}=0.7000 \\
\lambda_{22}=0.3000\end{array}$} \\
\hline & $\frac{1}{\sqrt{\operatorname{det}(P)}}$ & 0.0938 & 0.0938 & \\
\hline \multirow[t]{2}{*}{$p=0.8$} & $t$ & 0.3205 & 0.3205 & \multirow{2}{*}{$\begin{array}{l}\lambda_{11}=0.8000 \\
\lambda_{12}=0.2000 \\
\lambda_{21}=0.8000 \\
\lambda_{22}=0.2000\end{array}$} \\
\hline & $\frac{1}{\sqrt{\operatorname{det}(P)}}$ & 0.0715 & 0.0715 & \\
\hline \multirow[t]{2}{*}{$p=0.9$} & $t$ & 0.1803 & 0.1803 & \multirow{2}{*}{$\begin{array}{l}\lambda_{11}=0.9000 \\
\lambda_{12}=0.1000 \\
\lambda_{21}=0.9000 \\
\lambda_{22}=0.1000\end{array}$} \\
\hline & $\frac{1}{\sqrt{\operatorname{det}(P)}}$ & 0.0402 & 0.0402 & \\
\hline
\end{tabular}



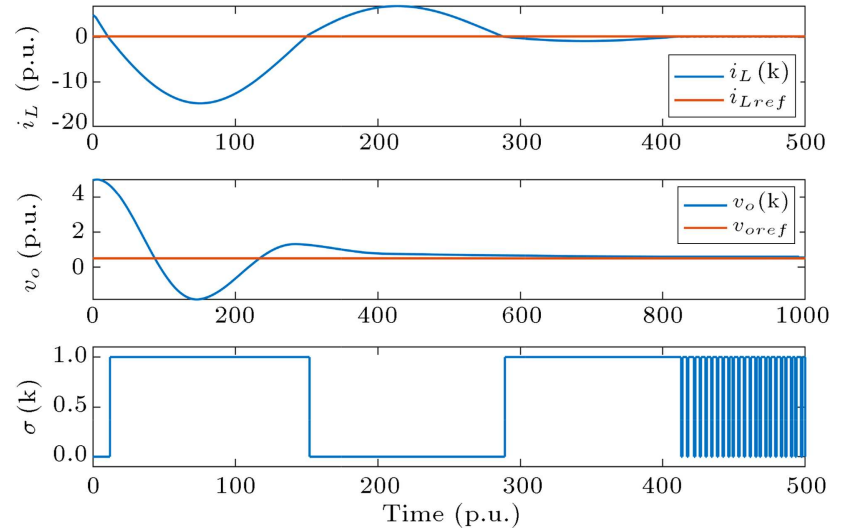

Figure 12. Time evolution of the state variables and switching function for the DC-DC buck converter.

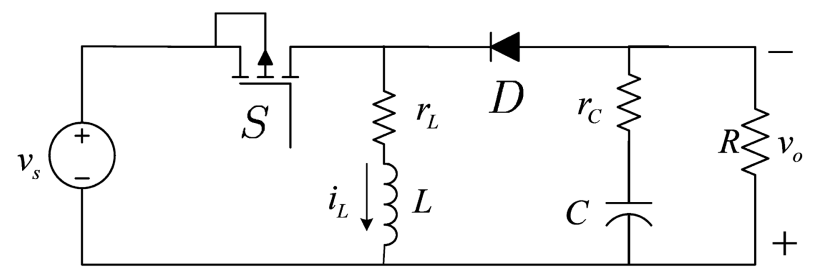

Figure 13. DC-DC buck-boost converter.

Eq. (58) where [62]:

$$
\begin{aligned}
& A_{c 1}=\left[\begin{array}{cc}
-\frac{r_{L}}{L} & 0 \\
0 & -\frac{1}{C} \frac{1}{\left(R+r_{C}\right)}
\end{array}\right], \quad b_{c 1}=\left[\begin{array}{c}
\frac{v_{s}}{L} \\
0
\end{array}\right] \\
& A_{c 2}=\left[\begin{array}{cc}
\frac{R}{R+r_{C}}\left(\frac{1}{C}-\frac{r_{L}}{L}-\frac{1}{L} r_{L}\right) & -\frac{R}{R+r_{C}}\left(\frac{r_{C}}{L}+\frac{1}{R C}\right)
\end{array}\right], \\
& b_{c 2}=\left[\begin{array}{l}
0 \\
0
\end{array}\right] .
\end{aligned}
$$

Based on Table 6, except for the operating point $p=0.8$, the conditions in Theorem 3 of [20] yield less conservative results. The state trajectories as well as the invariant set of attraction corresponding to the operating point $p=0.5$ are shown in Figure 14 . Figure 15 illustrates the time evolutions of the state variables and switching sequence corresponding to the initial condition $i_{L}(0)=v_{o}(0)=5 p . u$.

In sum, according to foregoing results, one may conclude that the proposed conditions in Theorem 1 are more suitable in terms of implementation than Theorem 3 of [20]. Moreover, statistically and not in all cases, the conditions of Theorem 3 in [20] produce less conservative results since more unknown variables and higher degrees of freedom are considered in constructing the ultimate invariant set of attraction.

\section{Conclusion}

In this paper, globally practical stabilization of the discrete-time switched affine systems was proposed.

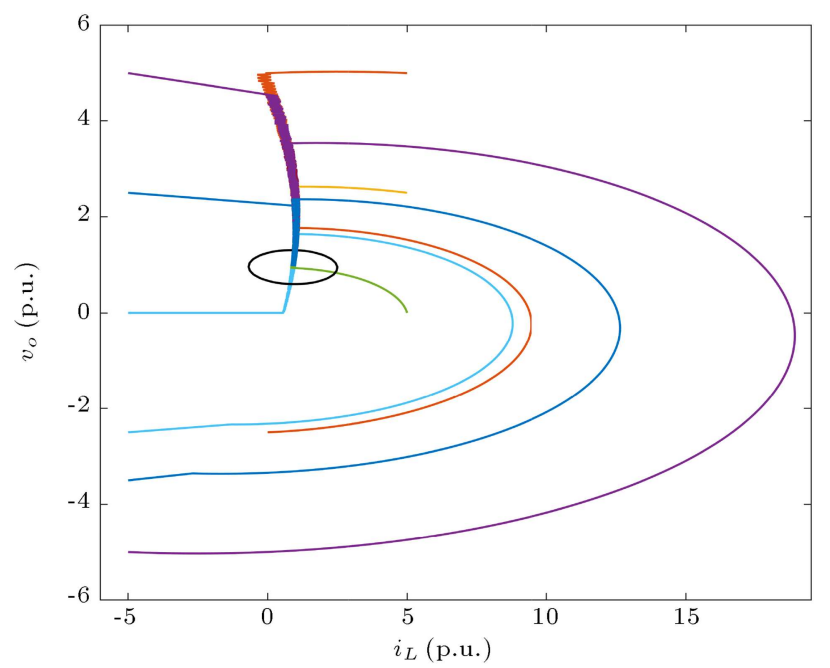

Figure 14. Invariant set of attraction and state trajectories of the DC-DC buck-boost converter.
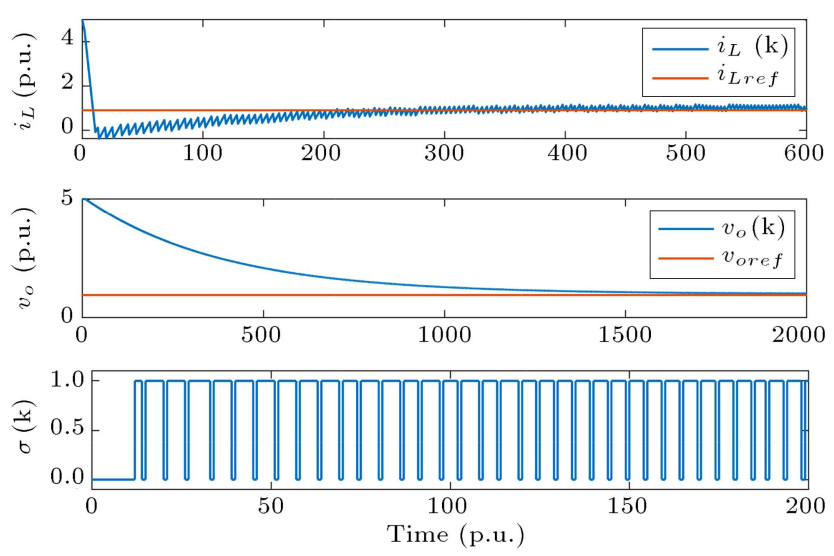

Figure 15. Time evolution of the state variables and switching function for the DC-DC buck-boost converter.

More specifically, in contrast to the existing two-stage stabilization methods, the proposed stability conditions were derived in a single stage based on a common quadratic Lyapunov function such that there was no need to compute a Schur stable matrix as a convex combination of operating modes in a preprocessing stage. Moreover, the equilibrium point around which the ultimate convergence set was constructed was not required to be a part of a specific set. The proposed stability conditions were formulated as a set of Bilinear Matrix Inequalities (BMIs) by considering some design criteria such as minimum size of the invariant set of attraction and smaller number of unknown variables. Finally, an academic example and various DC-DC converters were used to provide evidence for the merit of the proposed stabilization method.

Several research directions are suggested for further research. The first one is related to the use of more efficient and less conservative Lyapunov functions such as the structured Lyapunov functions proposed 
M. Hejri/Scientia Iranica, Transactions D: Computer Science \& ... 28 (2021) 1621-1642

Table 6. Ellipsoid size comparisons for the DC-DC buck-boost converter.

\begin{tabular}{|c|c|c|c|c|}
\hline $\begin{array}{c}\text { Operating } \\
\text { point }\end{array}$ & $\begin{array}{l}\text { Comparison } \\
\text { quantities }\end{array}$ & $\begin{array}{l}\text { Theorem } 1 \\
\text { ellipsoid size }\end{array}$ & $\begin{array}{l}\text { Theorem } 3 \text { of }[20] \\
\lambda_{1}=\frac{\lambda_{21}}{\sum_{i \in \mathbb{K}} \lambda_{2 i}} \\
\lambda_{2}=\frac{\lambda_{22}}{\sum_{i \in \mathbb{K}} \lambda_{2 i}} \\
\text { ellipsoid size }\end{array}$ & $\begin{array}{c}\text { Theorem } 1 \\
\sum_{i \in \mathbb{K}} \lambda_{1 i}=1 \\
\sum_{i \in \mathbb{K}} \lambda_{2 i}=1\end{array}$ \\
\hline \multirow[t]{2}{*}{$p=0.1$} & $t$ & 0.2558 & 0.2309 & \multirow{2}{*}{$\begin{array}{l}\lambda_{11}=0.0997 \\
\lambda_{12}=0.9003 \\
\lambda_{21}=0.0997 \\
\lambda_{22}=0.9003\end{array}$} \\
\hline & $\frac{1}{\sqrt{\operatorname{det}(P)}}$ & 0.0569 & 0.0516 & \\
\hline \multirow{2}{*}{$p=0.2$} & $t$ & 0.6061 & 0.5317 & \multirow{2}{*}{$\begin{array}{l}\lambda_{11}=0.1997 \\
\lambda_{12}=0.8003 \\
\lambda_{21}=0.1997 \\
\lambda_{22}=0.8003\end{array}$} \\
\hline & $\frac{1}{\sqrt{\operatorname{det}(P)}}$ & 0.1349 & 0.1188 & \\
\hline \multirow[t]{2}{*}{$p=0.3$} & $t$ & 1.0714 & 0.9290 & \multirow{2}{*}{$\begin{array}{l}\lambda_{11}=0.2997 \\
\lambda_{12}=0.7003 \\
\lambda_{21}=0.2997 \\
\lambda_{22}=0.7003\end{array}$} \\
\hline & $\frac{1}{\sqrt{\operatorname{det}(P)}}$ & 0.2386 & 0.2076 & \\
\hline \multirow[t]{2}{*}{$p=0.4$} & $t$ & 1.6919 & 1.4685 & \multirow{2}{*}{$\begin{array}{l}\lambda_{11}=0.3998 \\
\lambda_{12}=0.6002 \\
\lambda_{21}=0.3998 \\
\lambda_{22}=0.6002\end{array}$} \\
\hline & $\frac{1}{\sqrt{\operatorname{det}(P)}}$ & 0.3771 & 0.3283 & \\
\hline \multirow[t]{2}{*}{$p=0.5$} & $t$ & 2.5338 & 2.2281 & \multirow{2}{*}{$\begin{array}{l}\lambda_{11}=0.4999 \\
\lambda_{12}=0.5001 \\
\lambda_{21}=0.4999 \\
\lambda_{22}=0.5001\end{array}$} \\
\hline & $\frac{1}{\sqrt{\operatorname{det}(P)}}$ & 0.5654 & 0.4985 & \\
\hline \multirow[t]{2}{*}{$p=0.6$} & $t$ & 3.7061 & 3.3483 & \multirow{2}{*}{$\begin{array}{l}\lambda_{11}=0.6000 \\
\lambda_{12}=0.4000 \\
\lambda_{21}=0.6000 \\
\lambda_{22}=0.4000\end{array}$} \\
\hline & $\frac{1}{\sqrt{\operatorname{det}(P)}}$ & 0.8288 & 0.7499 & \\
\hline \multirow[t]{2}{*}{$p=0.7$} & $t$ & 5.3754 & 5.0887 & \multirow{2}{*}{$\begin{array}{l}\lambda_{11}=0.7001 \\
\lambda_{12}=0.2999 \\
\lambda_{21}=0.7001 \\
\lambda_{22}=0.2999\end{array}$} \\
\hline & $\frac{1}{\sqrt{\operatorname{det}(P)}}$ & 1.2073 & 1.1419 & \\
\hline \multirow[t]{2}{*}{$p=0.8$} & $t$ & 7.6283 & 7.8255 & \multirow{2}{*}{$\begin{array}{l}\lambda_{11}=0.8001 \\
\lambda_{12}=0.1999 \\
\lambda_{21}=0.8001 \\
\lambda_{22}=0.1999\end{array}$} \\
\hline & $\frac{1}{\sqrt{\operatorname{det}(P)}}$ & 1.7300 & 1.7659 & \\
\hline \multirow[t]{2}{*}{$p=0.9$} & $t$ & - & - & \multirow[t]{2}{*}{-} \\
\hline & $\frac{1}{\sqrt{\operatorname{det}(P)}}$ & - & - & \\
\hline
\end{tabular}


in [49]. The second suggestion is to focus on the local or global optimization algorithms by developing more efficient numerical algorithms in order to achieve better performance indices such as convergence, higher convergence speed, and better solutions in the sense of the closeness to the real global optimum point. Finally, the extension of the proposed stabilization technique to uncertain switched affine systems is another important research direction.

\section{References}

1. Lygeros, J. and Sastry, S. "Verified hybrid controllers for automated vehicles", IEEE Trans. Autom. Control, 43(4), pp. 522-539 (1998).

2. Torrisi, D.F. and Bemporad, A. "HYSDEL-a tool for generating computational hybrid models for analysis and synthesis problems", IEEE Trans. Control Syst. Technol., 12(2), pp. 235-249 (2004).

3. Bemporad, A. and Morari, M. "Control of systems integrating logic, dynamic and constraints", Automatica, 35(3), pp. 407-427 (1999).

4. Hejri, M., Giua, A., and Mokhtari, H. "On the complexity and dynamical properties of mixed logical dynamical systems via an automaton-based realization of discrete-time hybrid automaton", Int. J. of Robust Nonlin., 28(16), pp. 4713-4746 (2018).

5. Hejri, M. and Mokhtari, H. "On the well-posedness, equivalency and low-complexity translation techniques of discrete-time hybrid automaton and piecewise affine systems", Scientia Iranica, Transactions D, Computer Science \& Electrical Engineering (2019) (In Press). DOI: $10.24200 /$ sci.2019.53308.3177

6. Sun, Z. and Sam Ge, S., Switched Linear Systems, Control and Design, E.D. Sontag, M. Thoma, A. Isidori and J.H. van Schuppen, Eds., Springer-Verlag London (2005).

7. Liberzon, D., Switching in Systems and Control, T. Basar, Bikhauser Boston (2003).

8. Zhao, W., Kao, Y., Niu, B., et al., Control Synthesis of Switched Systems, Springer International Publishing Switzerland (2017).

9. Sun, Z. and Sam Ge, S., Stability Theory of Switched Dynamical Systems, A. Isidori, J.H. van Schuppen, E.D. Sontag, M. Thoma and M. Krstic, Eds., SpringerVerlag London (2011).

10. Liberzon, D. and Morse, A.S. "Basic problems in stability and design of switched systems", IEEE Control Syst. Mag. , 19(5), pp. 59-70 (1999).

11. Hai Lin, H. and Antsaklis, P.J. "Stability and stabilizability of switched linear systems: a survey of recent results", IEEE Trans. Autom. Control, 54(2), pp. 308322 (2009).

12. Decarlo, R.A., Branicky, M.S., Pettersson, S., et al. "Perspectives and results on the stability and stabilizability of hybrid systems", Proceedings of the IEEE, 88(7), pp. 1069-1082 (2000).
13. Shorten, R., Wirth, F., Mason, O., et al. "Stability criteria for switched and hybrid systems", SIAM Review, 49(4), pp. 545-592 (2007).

14. Deaecto, G.S., Geromel, J.C., Garcia, F.S., et al. "Switched affine systems control design with application to DC-DC converters", IET Control Theory A., 4(7), pp. 1201-1210 (2010).

15. Baldi, S., Papachristodoulou, A., and Kosmatopoulos, E.B. "Adaptive pulse width modulation design for power converters based on affine switched systems", Nonlinear Anal-Hybri., 30, pp. 306-322 (2018).

16. Yoshimora, V.L., Assuncao, E., Pires da Silva, E.R., et al. "Observer-based control design for switched affine systems and applications to DC\%.DC converters", Journal of Control, Automation and Electrical Systems, 24(4), pp. 535-543 (2013).

17. Corona, D., Buisson, J., De Schutter, B., et al. "Stabilization of switched affine systems: An application to the buck-boost converter", Proceedings of American Control Conf., New York, pp. 6037-6042 (2007).

18. Albea, C., Garcia, G., and Zaccarian, L. "Hybrid dynamic modeling and control of switched affine systems: application to DC-DC converters", IEEE 5 4th Annual Conf. on Decision and Control, Osaka, Japan, pp. 2264-2269 (2015).

19. Beneux, G., Riedinger, P., Daafouz, J., et al. "Adaptive stabilization of switched affine systems with unknown equilibrium points: application to power converters", Automatica, 99, pp. 82-91 (2019).

20. Deaecto, G.S. and Geromel, J.C. "Stability analysis and control design of discrete-time switched affine systems", IEEE Trans. Autom. Control, 62(8), pp. 4058-4065 (2017).

21. Egidio, L.N. and Deaecto, G.S. "Novel practical stability conditions for discrete-time switched affine systems", IEEE Trans. Autom. Control, 64(11), pp. 4705-4710 (2019).

22. Albea Sanchez, C., Garcia, G., Sabrina, H., et al. "Practical stabilisation of switched affine systems with dwell-time guarantees", IEEE Trans. Autom. Control, 64(11), pp. 4811-4817 (2019).

23. Hetel, L. and Fridman, E. "Robust sampled-data control of switched affine systems", IEEE Trans. Autom. Control, 58(11), pp. 2922-2928 (2013).

24. Hauroigne, P., Riedinger, P., and Iung, C. "Switched affine systems using sampled-data controllers: robust and guaranteed stabilization", IEEE Trans. Autom. Control, 56(12), pp. 2929-2935 (2011).

25. Kuiava, R., Ramos, R.A., Pota, H.R., et al. "Practical stability of switched systems without a common equilibria and governed by a time-dependent switching signal", Eur. J. Control, 19(3), pp. 206-213 (2013). 
26. Xu, X., Zhai, G., and He, S. "On practical asymptotic stabilizability of switched affine systems", Nonlinear Anal-Hybri., 2(1), pp. 196-208 (2008).

27. Xu, X. and Zhai, G. "Practical stability and stabilization of hybrid and switched systems", IEEE Trans. Autom. Control, 50(11), pp. 1897-1903 (2005).

28. Xu, X., Zhai, G., and He, S. "Stabilizability and practical stabilizability of continuous-time switched systems: a unified view", Proceedings of the 2007 American Control Conf., New York City, USA, pp. 663-668 (2007).

29. Xu, X., Zhai, G., and He, S. "Some results on practical stabilizability of discrete-time switched affine systems", Nonlinear Anal-Hybri., 4(1), pp. 113-121 (2010).

30. Xu, X., Zhai, G., and He, S. "On practical stabilizability of discrete-time switched affine systems", Joint 48th IEEE Conf. on Decision and Control, Shanghai, China, pp. 2144-2149 (2009).

31. Boyd, S., El Ghaoui, L., Feron, E., et al., Linear Matrix Inequalities in Systems and Control Theory, Society for Industrial and Applied Mathematics, SIAM (1994).

32. Duan, G.-R. and Yu, H.-H., LMIs in Control Systems: Analysis, Design and Applications, CRC Press, Taylor \& Francis Group (2013).

33. Blondel, V. and Tsitsiklis, J.N. "NP-Hardness of some linear control design problems", SIAM J. Control and Optim., 35(6), pp. 2118-2127 (1997).

34. Scharlau, C.C., de Oliveria, M.C., Trofino, A., et al. "Switching rule design for affine switched systems using a max-type composition rule", IEEE Trans. Autom. Control, 68, pp. 1-8 (2014).

35. Trofino, A., Assmann, D., Scharlau, C.C., et al. "Switching rule design for switched dynamic systems with affine vector fields", IEEE Trans. Autom. Control, 54(9), pp. 2215-2222 (2009).

36. Trofino, A., Scharlau, C.C., and Coutinho, D.F. "Corrections to "Switching rule design for switched dynamic systems with affine vector fields", IEEE Trans. Autom. Control, 57(4), pp. 1080-1082 (2014).

37. VanAntwerp, J.G. and Braatz, R.D. "A tutorial on linear and bilinear matrix inequalities", J. Process Contr., 10(4), pp. 365-385 (2000).

38. Hassibi, A., How, J., and Boyd, S. "A path-following method for solving BMI problems in control", Proceedings of the American Control Conf., San Diego, California, pp. 1385-1389 (1999).

39. Bolzern, P. and Spinelli, W. "Quadratic stabilization of a switched affine system about a nonequilibrium point", Proceedings of the 2004 American Control Conf., Boston, Massachusetts, pp. 3890-3895 (2004).
40. Deaecto, G.S. and Santos, G.C. "State feedback $H_{\infty}$ control design of continuous-time switched-affine systems", IET Control Theory A., 9(10), pp. 1511-1516 (2014).

41. Deaecto, G.S. "Dynamic output feedback $H_{\infty}$ control of continuous-time switched affine systems", Automatica, 71, pp. 44-49 (2016).

42. Poznyak, A., Polyakov, A., and Azhmyakov, V. "Attractive Ellipsoids in Robust Control", T. Basar, Ed., Birkhauser (2014).

43. Perez, C., Azhmyakov, V., and Poznyak, A. "Practical stabilization of a class of switched systems: dwell-time approach", IMA J. of Math. Control I., 32(4), pp. 689$702(2015)$.

44. Khalil, H., Nonlinear Systems, Prentice Hall, third edition (2003).

45. Lakshmikantham, V., Leela, S., and Martynyuk, A.A., Practical Stability of Nonlinear Systems, World Scientific (1990).

46. Geromel, J.C. and Colaneri, P. "Stability and stabilization of discrete-time switched systems", Int. J. Control, 79(7), pp. 719-728 (2006).

47. Lofberg, J. "YALMIP: A toolbox for modeling and optimization in MATLAB", IEEE International Symposium on Computer Aided Control Systems Design, Taipei, Taiwan, pp. 284-289 (2004).

48. Kocvara, M. and Stingl, M. PENBMI Users Guide (Version 2.1), www.penopt.com (2006).

49. Lacerda, M.J. and Gomide, T. da S. "Stability and stabilizability of switched discrete-time systems based on structured Lyapunov functions", IET Control Theory A., 14(5), pp. 781-789 (2020).

50. Patra, P., Patra, A., and Misra, N. "A singleinductor multiple-output switcher with simultaneous buck, boost, and inverted outputs", IEEE Trans. Power Electron., 27(4), pp. 1936-1951 (2012).

51. Patra, P., Ghosh, J., and Patra, A. "Control scheme for reduced cross-regulation in single-inductor multipleoutput DC-DC converters", IEEE Trans. Ind. Electron., 60(11), pp. 5095-5104 (2013).

52. Wang, B., Kanamarlapudi, V.R.K., Xiang, L., Peng, X., Tan, K.T., and So, P.L. "Model predictive voltage control for single-inductor multiple-output DC-DC converter with reduced cross regulation", IEEE Trans. Ind. Electron., 63(7), pp. 4187-4197 (2016).

53. Shen, Z., Chang, X., Wang, W., Tan, X., Yan, N., and Min, H. "Predictive digital current control of singleinductor multiple-output converters in CCM with low 
cross regulation", IEEE Trans. Power Electron., 27(4), pp. 1917-1925 (2012).

54. Dasika, J.D., Bahrani, B., Saeedifard, M., Karimi, A., and Rufer, A. "Multivariable control of singleinductor dual-output buck converters", IEEE Trans. Power Electron., 29(4), pp. 2061-2070 (2014).

55. Trevisan, D., Mattavelli, P., and Tenti, P. "Digital control of single-inductor multiple-output step-down DCDC converters in CCM", IEEE Trans. Ind. Electron., 55(9), pp. 3476-3483 (2008).

56. Senesky, M., Eirea, G., and KOO, T.J. "Hybrid modeling and control of power electronics", Hybrid Systems: Computations and Control, Lecture Notes in Computer Science, Springer, pp. 450-465 (2003).

57. Mariéthoz, S., Almér, S., Bâja M., et al. "Comparison of hybrid control techniques for buck and boost DCDC converters", IEEE Trans. Control Syst. Technol., 18(5), pp. 1126-1145 (2010).

58. Hejri, M. and Mokhtari, H. "Hybrid predictive control of a DC-DC boost converter in both continuous and discontinuous current modes of operations", Optim. Contr. Appl. Met., John Wiley and Sons, Inc., 32(3), pp. 270-284 (2011).

59. Hejri, M. and Mokhtari, H. "Hybrid modeling and control of a DC-DC boost converter via Extended Mixed Logical Dynamical systems (EMLDs)", IEEE 5th Power Electronics, Drive Systems and Technologies Conference (PEDSTC), pp. 373-378 (2014).

60. Hejri, M. and Mokhtari, H. "Global hybrid modeling and control of a buck converter: a novel concept", Int. J. Circ. Theor. App., John Wiley and Sons, Inc., 37(9), pp. 968-986 (2009).

61. Hejri, M. and Giua, A. "Hybrid modeling and control of switching DC-DC converters via MLD systems",
IEEE 7th International Conference on Automation Science and Engineering, Trieste, Italy, pp. 714-719 (2011).

62. Hejri, M. "Global hybrid modeling and control of a DC-DC buck-boost converter via mixed logical dynamical systems", Iranian Journal of Electrical and Computer Engineering, 17(1), pp. 1-12 (2019) (In Persian).

\section{Biography}

Mohammad Hejri received his BSc degree from Tabriz University in 2000 and the MSc degree from Sharif University of Technology, Tehran, Iran in 2002, both in Electrical Engineering. He received his $\mathrm{PhD}$ degree in Electrical Engineering from Sharif University of Technology, Tehran and the University of Cagliari, Cagliari, Italy, in 2010 as a co-tutorship program. He has been with several industries and research centers such as Iran Tractor Foundry Company, Azerbaijan Regional Electric Company, Tabriz Oil and Refining Company and Iran's Niroo (Energy) Research Institute (NRI). From 2010 to 2012, he was a Postdoctoral Research Associate at the Department of Electric Power and Energy Systems, School of Electrical Engineering, Royal Institute of Technology (KTH), Stockholm, Sweden. In 2012, he joined to the Department of Electrical Engineering, Sahand University of Technology, Tabriz where he is currently an Associate Professor. His research interests include control theory with applications in power electronics, renewable energy, and power systems. 